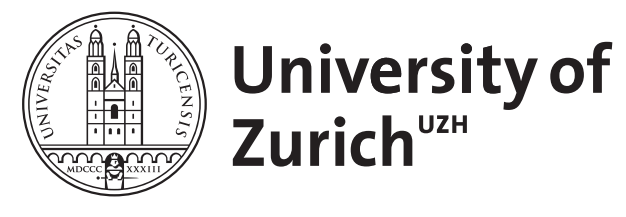

\title{
Synthesis of new Bis-imidazole derivatives
}

\author{
Jasiński, M ; Mlostoń, Grzegorz ; Mucha, P ; Linden, Anthony ; Heimgartner, H
}

\begin{abstract}
The reaction of aldimines with alpha-(hydroxyimino) ketones of type 10 (1,2-diketone monooximes) was used to prepare 2-unsubstituted imidazole 3-oxides 11 bearing an alkanol chain at N(1) (Scheme 2 , Table 1). These products were transformed into the corresponding $2 \mathrm{H}$-imidazol-2-ones 13 and $2 \mathrm{H}$ imidazole-2-thiones 14 by treatment with Ac2O and 2,2,4,4-tetramethylcyclobutane-1,3-dithione, respectively (Scheme 3). The three-component reaction of 10, formaldehyde, and an alkane-1,omega-diamine 15 gave the bis[1H-imidazole 3-oxides] 16 (Scheme 4, Table 2). With Ac2O, 2,2,4,4-tetramethylcyclobutane1,3-dithione or Raney-Ni, the latter reacted to give the corresponding bis[2H-imidazol-2-ones] 19 and 20 , bis[2H-imidazol-2-thione] 21, and bis[imidazole] 22, respectively (Schemes 5 and 6). The structures of $11 \mathrm{a}$ and $16 \mathrm{~b}$ were established by X-ray crystallography.
\end{abstract}

DOI: https://doi.org/10.1002/hlca.200790186

Posted at the Zurich Open Repository and Archive, University of Zurich ZORA URL: https://doi.org/10.5167/uzh-50665

Journal Article

Accepted Version

Originally published at:

Jasiński, M; Mlostoń, Grzegorz; Mucha, P; Linden, Anthony; Heimgartner, H (2007). Synthesis of new Bis-imidazole derivatives. Helvetica Chimica Acta, 90(9):1765-1780.

DOI: https://doi.org/10.1002/hlca.200790186 
Prof. Dr. H. Heimgartner

Tel. 0446354282

Fax 0446356812

e-mail: heimgart@oci.uzh.ch

\title{
Synthesis of New Bis-Imidazole Derivatives
}

\author{
by Marcin Jasinski ${ }^{1)}$, Grzegorz Mloston*, and Paulina Mucha ${ }^{2)}$ \\ University of Lódz, Department of Organic and Applied Chemistry, Narutowicza 68, \\ PL-90-136 Lódz \\ (phone: +48 42635 5761; fax: +48 42635 5380; e-mail: gmloston@ @uni.lodz.pl)
}

and Anthony Linden and Heinz Heimgartner*

Organisch-chemisches Institut der Universität Zürich, Winterthurerstrasse 190, CH-8057 Zürich

(phone: +41 44635 4282; fax: +41 44635 6812; e-mail: heimgart@ oci.uzh.ch)

${ }^{1}$ ) Part of the planned Ph. D. thesis of M. J., University of Lódz.

${ }^{2}$ ) Part of the planned Ph. D thesis of P. M., University of Lódz. 
The reaction of aldimines with $\alpha$-(hydroxyimino)ketones of type $\mathbf{1 0}$ (1,2-diketone monooximes) was used to prepare 2-unsubstituted imidazole 3-oxides $\mathbf{1 1}$ bearing a functionalized alkyl residue at $\mathrm{N}(1)$. These products were transformed into the corresponding imidazole-2-ones $\mathbf{1 3}$ and imidazole-2-thiones $\mathbf{1 4}$ by treatment with $\mathrm{Ac}_{2} \mathrm{O}$ and 2,2,4,4-tetramethylcyclobutane-1,3-dithione, respectively. The three-component reaction of 10, formaldehyde, and an alkane-1, $\omega$-diamine 15 gave the bis-(imidazole 3oxides) 16. With $\mathrm{Ac}_{2} \mathrm{O}, 2,2,4,4$-tetramethylcyclobutane-1,3-dithione or Raney-Ni, the latter reacted to give the corresponding bis-(imidazol-2(3H)-ones) $\mathbf{1 9}$, bis-(imidazol2(3H)-thiones) 20, and bis-imidazoles $\mathbf{2 2}$, respectively. The structures of $\mathbf{1 1 a}$ and $\mathbf{1 6 \mathbf { b }}$ were established by X-ray crystallography. 


\section{Introduction. -}

The importance of imidazole and benzimidazole derivatives both in the field of biologically active compounds and in organic synthesis is well documented (see for example [1-9]). Complexes of imidazoles [10] and imidazole derived carbenes [11] with diverse metal cations have also been studied extensively. In a series of recent papers, the synthesis of 2-unsubstituted imidazole $N$-oxides was reported [12-15]. These derivatives were shown to be useful starting materials for the preparation of other imidazoles, such as imidazole-2-thiones, imidazole-2-carbonitriles, imidazol-2-ones, and $\mathrm{N}$-alkyl or $\mathrm{N}$-arylimidazol-2-amines. An important feature of the structure of imidazole $\mathrm{N}$-oxides $\mathbf{1}$ is their similarity with nitrones, which are well known 1,3-dipoles applied for the synthesis of N,O-containing five-membered heterocycles [16]. In the case of the electron-deficient 2,2-bis(trifluoromethyl)ethene-1,1-dicarbonitrile (BTF, 2), the reaction with 2-unsubstituted imidazole-3-oxides $\mathbf{1}$ led to imidazol-2ylidenepropanedinitriles 3 and hexafluoroacetone. The formation of these products occurred via a regioselective [2+3]-cycloaddition to give $\mathbf{4}$ and subsequent fragmentation to produce $\mathbf{3}$ (Scheme 1). The reaction was proposed to proceed stepwise via a zwitterionic intermediate 5, which, in the presence of $\mathrm{H}_{2} \mathrm{O}$, underwent the conversion to imidazol-2-ones [15].

\section{Scheme 1}

A similar reaction pattern is observed when perfluoropropene is used as a dipolarophile. After elimination of carbonic difluoride $\left(\mathrm{F}_{2} \mathrm{C}=\mathrm{O}\right)$, the labile cycloadduct of type $\mathbf{4}$ gives the corresponding 2-(1,2,2,2-tetrafluoroethyl)imidazole [17]. 
Recently, new approaches for the synthesis of both imidazoles and imidazole $\mathrm{N}$ oxides were published [18] [19]. Taking into account that imidazole $N$-oxides can easily be deoxygenated, the second approach opens access to a larger number of differently functionalized derivatives. Considering the type of starting materials applied in the syntheses of imidazole $\mathrm{N}$-oxides, oximes and mono-oximes of 1,2-dicarbonyl compounds ( $\alpha$-(hydroxyimino)ketones) are of special interest. In the latter case, the synthesis can be performed with an aldimine or, alternatively, in a three-component reaction with a primary amine and an aldehyde. However, it has been reported that in the three-component reaction with formaldehyde, isomerization of the initially formed imidazole $\mathrm{N}$-oxide to the corresponding imidazol-2-one already takes place in the reaction mixture [20].

As a continuation of our studies on imidazoles and imidazole $N$-oxides, the preparation of imidazole $N$-oxides bearing functionalized side chains should be elaborated. The only report on optically active imidazole $N$-oxides presented a method based on the use of $\alpha$-amino acids as the amino component in the three-component reaction [21]. However, racemization during the formation of the imidazole ring was a serious problem, and in some cases, only completely racemized products were obtained. In a very recent paper, chiral $\beta$-aminoalcohols with the stereogenic centre in the $\alpha$ - or $\beta$ position were reacted with glyoxal, formaldehyde, and ammonia to give optically active imidazoles of type 6 and 7 [9]. In another paper, the same method is reported for the synthesis of bis-imidazole 8. The authors claim that by using $(R, R)$-and $(S, S)-1,2-$ diphenylethane-1,2-diamine the $(R, R)$ and $(S, S)$ enantiomer of $\mathbf{8}$, respectively, was obtained. However, no data regarding their optical activity or $e, e$-values are given [18].

\section{Formulae 6, 7, and $\mathbf{8}$}


To the best of our knowledge, there are no known reports on the preparation of imidazoles and bis-(imidazole $N$-oxides) by using aminoalcohols and diamines, respectively, in reactions with $\alpha$-(hydroxyimino)ketones. In the present paper, we describe first results of this approach.

\section{Results and Discussion. -}

2.1. Preparation of 1-(Hydroxyalkyl)imidazole 3-Oxides. In analogy to the already described preparations of 1,3,5-tris-(2-hydroxyethyl)- and 1,3,5-tris-(3-hydroxypropyl)perhydro-1,3,5-triazines (12a and 12b, resp.) [22], compounds $\mathbf{1 2 c}, \mathbf{1 2 d}$ and $\mathbf{1 2 e}$ were obtained by treatment of the corresponding aminoalcohols with paraformaldehyde in $\mathrm{MeOH}$. The crude products were used for the condensation with $\alpha$ (hydroxyimino)ketones $\mathbf{1 0}$ in refluxing $\mathrm{EtOH}$ (Scheme 2). Under these conditions, perhydrotriazines $\mathbf{1 2}$ are known to undergo dissociation [12], and the monomeric formaldehyde imines reacted with $\mathbf{1 0}$ to give imidazole 3-oxides $\mathbf{1 1}$ (Table 1) according to the known mechanism (cf. [23]).

\section{Scheme 2}

Table 1. Imidazole 3-Oxides 11 Prepared from 10 and 12.

All imidazole 3-oxides $\mathbf{1 1}$ were characterized by their spectroscopic and analytical data. In most cases, the crystalline products contained variable amounts of $\mathrm{H}_{2} \mathrm{O}$. The ${ }^{1} \mathrm{H}-\mathrm{NMR}$ spectra show a characteristic down-field shifted signal for $\mathrm{H}-\mathrm{C}(2)$ at 
8.2-8.5 ppm. Furthermore, the structure of $11 \mathrm{a}$ was established by X-ray crystallography (Fig.1).

Fig. 1. ORTEP-Plot [24] of the molecular structure of 11a (arbitrary numbering of the atoms, $50 \%$ probability ellipsoids, the $\mathrm{H}_{2} \mathrm{O}$ molecule is not shown)

The asymmetric unit contains one zwitterionic molecule and half of a $\mathrm{H}_{2} \mathrm{O}$ molecule, which sits on a $C_{2}$-axis. The hydroxy group forms an intermolecular H-bond with the oxide $\mathrm{O}$-atom of a neighboring molecule and thereby links the molecules into extended chains which run in the $\left[\begin{array}{lll}1 & -1 & 0\end{array}\right]$ and $\left[\begin{array}{lll}1 & 1 & 0\end{array}\right]$ directions and can be described by a graph set motif [25] of $\mathrm{C}(8)$. Each $\mathrm{H}_{2} \mathrm{O}$ molecule forms two intermolecular $\mathrm{H}$ bonds with the oxide O-atoms of two $C_{2}$-related zwitterionic molecules, thereby crosslinking the two directions of the extended chains into two-dimensional networks, which lie parallel to the (001) plane.

According to [14], treatment of imidazole 3-oxides of type 11 in $\mathrm{CH}_{2} \mathrm{Cl}_{2}$ with $\mathrm{Ac}_{2} \mathrm{O}$ led to their isomerization to imidazol-2-ones, even at $0-5^{\circ}$. Heating of solutions of imidazol-2-ones in $\mathrm{Ac}_{2} \mathrm{O}$ resulted in the acetylation of $\mathrm{N}(3)$ [14]. In the case of 11b,c, and $\mathbf{e}$, the reaction with excess $\mathrm{Ac}_{2} \mathrm{O}$ at $0^{\circ}$ to room temperature resulted not only in the isomerization but also in acetylation of the $\mathrm{OH}$ group to yield the corresponding acetates 13 (Scheme 3). In none of the cases was acetylation of N(3) observed. In the IR as well as in the ${ }^{13} \mathrm{C}$-NMR spectra, the presence of an ester and a urea $\mathrm{C}=\mathrm{O}$ group is evidenced by absorptions at $c a .1740$ and $1670 \mathrm{~cm}^{-1}$ and 170 and $155 \mathrm{ppm}$, respectively. 


\section{Scheme 3}

In analogy to previously reported transformations of 2-unsubstituted imidazole 3-oxides [12], compounds 11a and 11e, respectively, reacted easily with 2,2,4,4tetramethylcyclobutane-1,3-dithione to give the corresponding imidazole-2-thiones $\mathbf{1 4 a}$ and $\mathbf{1 4 b}$ in high yield (Scheme 3).

Due to the fact that bis-imidazoles are of interest in the field of coordination chemistry [11], reactions of alkanediamines $\mathbf{1 5}$ with aldehydes and $\mathbf{1 0}$ were carried out in refluxing EtOH. In the case of formaldehyde used in excess (2.5 equiv.), the threecomponent reaction yielded 2-unsubstituted products $\mathbf{1 6}$ with a variable length of the aliphatic chain, which connects the two imidazole residues (Scheme 4, Table 2). The reaction with ethane-1,2-diamine $(\mathbf{1 5 a}), \mathbf{1 0 b}$, and acetaldehyde gave the expected bis(imidazole 3-oxide) $\mathbf{1 6 c}$ with the Me group at $\mathrm{C}(2)$ of the imidazole.

\section{Scheme 4}

Table 2. Prepared Bis-(imidazole 3-Oxides) 16.

The spectroscopic data confirm the structures of the bis-(imidazole 3-oxides) $\mathbf{1 6}$, and in the case of $\mathbf{1 6 b}$, the structure has been established by X-ray crystallography (Fig. 2). The heterocyclic molecule has crystallographic $C_{2}$ symmetry. The asymmetric unit includes a $\mathrm{H}_{2} \mathrm{O}$ molecule in a general position so that the ratio of heterocyclic molecules to $\mathrm{H}_{2} \mathrm{O}$ is $1: 2$. The two oxide $\mathrm{O}$-atoms in each bis-imidazole molecule are bridged by a pair of intermolecular H-bonds from each of two $\mathrm{H}_{2} \mathrm{O}$ molecules. Thus, two $\mathrm{H}_{2} \mathrm{O}$ 
molecules and the two oxide O-atoms from a single heterocyclic molecule combine to form a $C_{2}$-symmetric H-bonded loop with a graph set motif of $\mathrm{R}^{2}{ }_{4}(8)$.

Fig. 2. ORTEP-Plot [24] of the molecular structure of $\mathbf{1 6 b}$ (arbitrary numbering of the atoms, $50 \%$ probability ellipsoids, the $\mathrm{H}_{2} \mathrm{O}$ molecule is not shown)

It is worth mentioning that the attempted synthesis of a 1-(2aminoethyl)imidazole 3-oxide from 15a, formaldehyde, and 10a in a ratio of 1:1:1 did not afford the expected product and, again, 16a was obtained. As an alternative method, monoacetylated diamines $\mathbf{1 7}$ were reacted with formaldehyde, and the crude imines obtained thereby were heated together with $\mathbf{1 0}$ in EtOH. Under these conditions, imidazole 3-oxides of type $\mathbf{1 8}$ were formed and isolated as crystalline materials (Scheme 4).

The reactivity of bis-(imidazole 3-oxides) of type $\mathbf{1 6}$ toward $\mathrm{Ac}_{2} \mathrm{O}$ was tested using 16a. The isomerization to give the bis-(imidazole-2-one) 19 was achieved by heating 16a in a 1:1 mixture of $\mathrm{CHCl}_{3}$ and $\mathrm{Ac}_{2} \mathrm{O}$ under reflux for $3 \mathrm{~h}$ (Scheme 5). On the other hand, heating of $\mathbf{1 6 a}$ in boiling $\mathrm{Ac}_{2} \mathrm{O}$ resulted in complete acetylation of the rearranged product, and 20a was obtained in fair yield (Scheme 5). The analogous reaction sequence was observed with $\mathbf{1 6 e}$ and $\mathbf{1 6 f}$.

\section{Scheme 5}

The conversion of 2-unsubstituted imidazole 3-oxides to imidazole-2-thiones using 2,2,4,4-tetramethylcyclobutane-1,3-dithione (cf. Scheme 3) was applied efficiently 
in the case of 16e. The reaction was carried out in $\mathrm{CHCl}_{3}$ at room temperature, and the bis-(imidazole-2-thione) 21 precipitated from the solution (Scheme 6).

\section{Scheme 6}

Finally, the deoxygenation of $\mathbf{1 6 f}$ with Raney-Ni in EtOH led to the bisimidazole derivative $\mathbf{2 2}$ (Scheme 6).

\section{Conclusion. -}

The present study shows that the synthesis of 2-unsubstituted imidazole 3-oxides based on the three-component reaction of an $\alpha$-(hydroxyimino)ketone, formaldehyde, and an amino component is a convenient and efficient access to new derivatives derived from aminoalcohols and diamines. In the case of enantiomerically pure aminoalcohols, optically active products are formed without any racemization. The readily available bis-imidazole derivatives obtained from aliphatic $\alpha, \omega$-diamines are attractive ligands for the preparation of new metal complexes.

We thank the analytical sections of our institutes for spectra and analyses. G. $M$. acknowledges financial support by the Ministry of Science and Higher Education (Grant No. PBZ-KBN-126/T09/12), and H. H. thanks F. Hoffmann-La Roche AG, Basel, for financial support. 


\section{Experimental Part}

1. General. M.p.: Melt-Temp. II apparatus (Aldrich), in capillary; uncorrected. IR: NEXUS FT-IR spectrophotometer, in $\mathrm{KBr}$; in $\mathrm{cm}^{-1} \cdot{ }^{1} \mathrm{H}-$ and ${ }^{13} \mathrm{C}-\mathrm{NMR}$ : Tesla BS567A (80 and $20 \mathrm{MHz}$, resp.) or Bruker AC 300 instrument (300 and $75.5 \mathrm{MHz}$, resp.), in $\mathrm{CDCl}_{3}, \mathrm{CD}_{3} \mathrm{OD}$ or $\left(\mathrm{D}_{6}\right) \mathrm{DMSO}$; TMS as an internal standard. The multiplicity of the ${ }^{13} \mathrm{C}-\mathrm{NMR}$ signals was deduced from the DEPT spectra. MS (EI, ESI or CI): Finnigan MAT-90 or Finnigan SSQ-700 instruments. Elemental analyses were performed in the Analytical Laboratory of the University of Zürich.

2. Starting materials. $\alpha$-(Hydroxyimino)ketones $\mathbf{1 0}$ were obtained according to known protocols: butane-2,3-dione monooxime (10a) [26a], 1-phenylpropane-1,2-dione 1-oxime (10b) [26b] and 1-phenylpropane-1,2-dione 2-oxime (10c) [26c] by nitrosation of the corresponding ketones using isoamyl nitrate, 1,2-diphenylethane-1,2-dione monooxime (benzil monooxime, 10d) [26d] from dibenzoyl (benzil) and hydroxylamine hydrochloride.

3. Preparation of 1,3,5-Trisubstituted-1,2,3,4,5,6-hexahydro-1,3,5-triazines 12. Prepared analogously to a known protocol [22] from the following amines: 2aminoethan-1-ol (9a), 3-aminopropan-1-ol (9b), 1-aminopropan-2-ol (9c), 2aminobutan-1-ol ((RS)-9d), (+)-(S)-1-aminopropan-2-ol $((S)-9 \mathbf{c})$, and (+)-(1S,2S)-2amino-1-phenylpropane-1,3-diol $((S, S)-9 \mathbf{e})$, respectively. In a standard procedure, to the soln. of the corresponding aminoalcohol $(0.10 \mathrm{~mol})$ in $\mathrm{MeOH}(100 \mathrm{ml}), 0.11 \mathrm{~mol}$ of paraformaldehyde was added and the resulting suspension was stirred overnight. Then, the excess of paraformaldehyde was filtered and the filtrate was concentrated in vacuo to give $\mathbf{1 2}$ as a colorless or pale yellow oil in quantitative yield. The crude products $\mathbf{1 2}$ were used in the next step without purification. 
4. Synthesis of 1-Hydroxyalkylimidazole 3-Oxides 11. A soln. of $10 \mathrm{mmol}$ of dione monooxime (10a-d) and $12 \mathrm{mmol}$ of the corresponding 12 in abs. EtOH was heated for $3 \mathrm{~h}$. After evaporation of the solvent under reduced pressure, the resulting oil was washed twice with $\mathrm{Et}_{2} \mathrm{O}$, treated with acetone, and cooled. The product was obtained as a white solid, usually as a hydrate with a variable amount of $\mathrm{H}_{2} \mathrm{O}$. Samples for analysis were recrystallized from appropriate solvents. In the case of $\mathbf{1 1 e}, \mathbf{g}$, and $\mathbf{l}$, the resulting mixtures were purified by column chromatography (CC).

1-(2-Hydroxyethyl)-4,5-dimethyl-1H-imidazole 3-Oxide (11a). Yield $\left.{ }^{3}\right): 145 \mathrm{mg}$ (88\%). Colorless solid. M.p. 106-107 $\left(\mathrm{CHCl}_{3} /\right.$ hexane). IR: 3470-2700vs (br.), 1626m, $1447 m, 1404 s, 1386 m, 1346 s, 1324 s, 1194 m, 1149 m, 1092 s, 1075 s, 830 m, 755 m, 630 s$, 607s. ${ }^{1} \mathrm{H}-\mathrm{NMR}\left(\mathrm{CDCl}_{3}\right): 7.95(s, \mathrm{H}-\mathrm{C}(2)) ; 3.88,3.85\left(2 t, 2 \mathrm{CH}_{2}\right) ; 2.14,2.11(2 s, 2 \mathrm{Me})$. ${ }^{13} \mathrm{C}-\mathrm{NMR}\left(\mathrm{CDCl}_{3}\right): 125.9,121.6(2 s, \mathrm{C}(4), \mathrm{C}(5)) ; 125.8(d, \mathrm{C}(2)) ; 60.3,48.5(2 t, 2$ $\left.\mathrm{CH}_{2}\right)$; 8.7, $7.1(2 q, 2 \mathrm{Me})$. CI-MS: $157\left(17,[M+1]^{+}\right), 141$ (100). Anal. calc. for $\mathrm{C}_{7} \mathrm{H}_{12} \mathrm{~N}_{2} \mathrm{O}_{2} \cdot 0.5 \mathrm{H}_{2} \mathrm{O}$ (165.20): C 50.90, H 7.93, N 16.96; found C 50.88, H 7.50, N 17.08.

Suitable crystals for an X-ray crystal structure determination were grown from $\mathrm{CHCl}_{3} /$ hexane by slow evaporation of the solvent at r.t.

1-(2-Hydroxyethyl)-5-methyl-4-phenyl-1H-imidazole 3-Oxide (11b). Yield $\left.{ }^{3}\right)$ : $177 \mathrm{mg}$ (81\%). Colorless crystals. M.p. 186-188 $\left(\mathrm{CHCl}_{3} /\right.$ hexane). IR: 3350-2800vs (br.), 1614m, 1442m, 1389m, 1382m, 1353s, 1249m, 1213m, 1055vs, 770s, 716s, 699s, 608s. ${ }^{1} \mathrm{H}-\mathrm{NMR}\left(\mathrm{CDCl}_{3}\right): 8.27(s, \mathrm{H}-\mathrm{C}(2)) ; 7.55-7.34(m, 5$ arom. $\mathrm{H}) ; 4.05,3.84(2 t, 2$ $\left.\mathrm{CH}_{2}\right) ; 2.27(s, \mathrm{Me}) .{ }^{13} \mathrm{C}-\mathrm{NMR}\left(\mathrm{CDCl}_{3}\right): 129.7,128.7,128.3(3 d, 5$ arom. $\mathrm{CH}) ; 129.4(s$, arom. $\left.\mathrm{C}_{\mathrm{q}}\right) ; 127.0,126.2(2 s, \mathrm{C}(4), \mathrm{C}(5)) ; 123.4(d, \mathrm{C}(2)) ; 59.8,48.5\left(2 t, 2 \mathrm{CH}_{2}\right) ; 9.2$ (q,

3) Yield before crystallization. 
Me). EI-MS: $218\left(12, M^{+\cdot}\right), 202$ (100), 158 (46), 103 (56), 77 (27). Anal. calc. for $\mathrm{C}_{12} \mathrm{H}_{14} \mathrm{~N}_{2} \mathrm{O}_{2}$ (218.26): C 66.04, H 6.47, N 12.84; found C 66.15, H 6.35, N 12.42. 1-(2-Hydroxyethyl)-4,5-diphenyl-1H-imidazole 3-Oxide (11c). Yield $\left.{ }^{3}\right): 251 \mathrm{mg}$ (89\%). Colorless solid. M.p. 197-198 $\left(\mathrm{CHCl}_{3} /\right.$ petroleum ether). IR: 3350-2600vs (br.), $1443 m, 1393 m, 1350 s, 1200 m, 1076 m, 844 m, 772 s, 753 v s, 694 s, 658 m .{ }^{1} \mathrm{H}-\mathrm{NMR}$ $\left(\mathrm{CDCl}_{3}\right): 8.46(s, \mathrm{H}-\mathrm{C}(2)) ; 7.49-7.23(m, 10$ arom. $\mathrm{H}) ; 4.01,3.71\left(2 t, 2 \mathrm{CH}_{2}\right) .{ }^{13} \mathrm{C}-\mathrm{NMR}$ $\left(\mathrm{CDCl}_{3}\right): 130.7,129.7,129.5,129.0,128.5,128.0(6 d, 10$ arom. $\mathrm{CH}) ; 129.8,127.7$, 126.4, $125.7\left(4 s, 2\right.$ arom. $\left.\mathrm{C}_{\mathrm{q}}, \mathrm{C}(4), \mathrm{C}(5)\right) ; 127.6(d, \mathrm{C}(2)) ; 59.9,48.6\left(2 t, 2 \mathrm{CH}_{2}\right)$. EIMS: $280\left(31, M^{+}\right), 264(100), 219(48), 165(68), 103$ (71), 77 (57). Anal. calc. for $\mathrm{C}_{17} \mathrm{H}_{16} \mathrm{~N}_{2} \mathrm{O}_{2} \cdot 0.1 \mathrm{H}_{2} \mathrm{O}$ (282.13): C 72.37, H 5.79, N 9.93; found C 72.34, H 5.71, N 9.64 .

1-(2-Hydroxyethyl)-4-methyl-5-phenyl-1H-imidazole 3-Oxide (11d). Yield $\left.{ }^{3}\right)$ : $120 \mathrm{mg}$ (55\%). Colorless solid. M.p. 165-167 (acetone). IR: 3250-2550vs (br.), $1501 m, 1444 m, 1395 s, 1380 m, 1337 v s, 1165 s, 1080 s, 871 m, 757 s, 703 s, 646 m .{ }^{1} \mathrm{H}-$ $\operatorname{NMR}\left(\mathrm{CDCl}_{3}\right): 8.36(s, \mathrm{H}-\mathrm{C}(2)) ; 7.56-7.42(m, 5$ arom. $\mathrm{H}) ; 4.02,3.62\left(2 t, 2 \mathrm{CH}_{2}\right) ; 2.15$ $(s, \mathrm{Me}) .{ }^{13} \mathrm{C}-\mathrm{NMR}\left(\mathrm{CDCl}_{3}\right): 130.8,129.6,129.3(3 d, 5$ arom. $\mathrm{CH}) ; 127.8,127.4,126.8$ $\left(3 s\right.$, arom. $\left.\mathrm{C}_{\mathrm{q}}, \mathrm{C}(4), \mathrm{C}(5)\right) ; 60.5,49.1\left(2 t, 2 \mathrm{CH}_{2}\right) ; 7.6(q, \mathrm{Me})$. EI-MS: $218\left(76, M^{+\cdot}\right)$, 202 (100), 130 (63), 104 (72). Anal. calc. for $\mathrm{C}_{12} \mathrm{H}_{14} \mathrm{~N}_{2} \mathrm{O}_{2}$ (218.26): C 66.04, H 6.47, N 12.84; found C 66.38, H 6.43, N 12.46

1-(3-Hydroxypropyl)-5-methyl-4-phenyl-1H-imidazole 3-Oxide (11e). Yield after $\mathrm{CC}\left(\mathrm{R}_{\mathrm{f}}=0.59, \mathrm{SiO}_{2}, \mathrm{AcOEt} / \mathrm{MeOH}\right.$ 6:4): $218 \mathrm{mg}$ (94\%). Colorless solid. M.p. 140-142 (acetone). IR: 3350-2550vs (br.), 1497m, 1467m, 1400m, 1363s, 1344s, 1315m, 1253s, $1231 m, 1064 s, 930 m, 766 s, 712 s, 702 s, 695 s, 608 s .{ }^{1} \mathrm{H}-\mathrm{NMR}\left(\mathrm{CD}_{3} \mathrm{OD}\right): 8.31(s, \mathrm{H}-$ $\mathrm{C}(2))$; 7.59-7.40 (m, 5 arom. H); 4.14, $3.59\left(2 t, 2 \mathrm{CH}_{2}\right) ; 2.29(s, \mathrm{Me}) ; 1.99\left(m, \mathrm{CH}_{2}\right)$. ${ }^{13} \mathrm{C}-\mathrm{NMR}\left(\mathrm{CD}_{3} \mathrm{OD}\right): 131.1,129.8,129.5(3 d, 5$ arom. $\mathrm{CH}) ; 130.6,128.2,125.2(3 s$, 
arom. $\left.\mathrm{C}_{q}, \mathrm{C}(4), \mathrm{C}(5)\right) ; 127.8(d, \mathrm{C}(2))$; 58.9, 44.0, $33.7\left(3 t, 3 \mathrm{CH}_{2}\right)$; $9.2(q$, Me). EI-MS: $232\left(5, M^{+}\right), 214(100), 117$ (47), 55 (49). Anal. calc. for $\mathrm{C}_{13} \mathrm{H}_{16} \mathrm{~N}_{2} \mathrm{O}_{2}$ (232.28): C 67.22, H 6.94, N 12.06; found C 67.46, H 7.06, N 11.70.

1-(3-Hydroxypropyl)-4,5-diphenyl-1H-imidazole 3-Oxide (11f). Yield $\left.{ }^{3}\right): 140 \mathrm{mg}$ (47\%). Colorless solid. M.p. 191-193º (acetone). IR: 3350-2650vs (br.), 1445m, 1392s, $1345 s, 1198 m, 1078 s, 769 s, 756 s, 698 s, 657 m .{ }^{1} \mathrm{H}-\mathrm{NMR}\left(\mathrm{CD}_{3} \mathrm{OD}\right): 8.51(s, \mathrm{H}-\mathrm{C}(2))$; 7.48-7.26 (m, 10 arom. $\mathrm{H}) ; 4.09,3.48\left(2 t, 2 \mathrm{CH}_{2}\right) ; 1.81\left(m, \mathrm{CH}_{2}\right) .{ }^{13} \mathrm{C}-\mathrm{NMR}\left(\mathrm{CD}_{3} \mathrm{OD}\right)$ : 132.1, 131.2, 130.9, 130.3, 129.7, 129.2, 128.9 (7d, 10 arom. $\mathrm{CH}, \mathrm{C}(2))$; 131.4, 129.3, 128.5, $127.8\left(4 s, 2\right.$ arom. $\left.\mathrm{C}_{\mathrm{q}}, \mathrm{C}(4), \mathrm{C}(5)\right)$; 59.0, 44.8, $33.8\left(3 t, 3 \mathrm{CH}_{2}\right)$. EI-MS: 294 (7, $M^{+}$), 276 (75), 165 (19), 117 (100), 104 (19), 77 (22). Anal. calc. for $\mathrm{C}_{18} \mathrm{H}_{18} \mathrm{~N}_{2} \mathrm{O}_{2} \cdot 0.25$ $\mathrm{H}_{2} \mathrm{O}$ (298.86): C 72.34, H 6.24, N 9.37; found C 72.26, H 6.08, N 9.30.

1-(3-Hydroxypropyl)-4-methyl-5-phenyl-1H-imidazole 3-Oxide (11g). Yield after $\mathrm{CC}\left(\mathrm{R}_{\mathrm{f}}=0.65, \mathrm{SiO}_{2}, \mathrm{AcOEt} / \mathrm{MeOH} 1: 1\right): 153 \mathrm{mg}$ (66\%). Colorless solid. M.p. 139-140 $\left(\mathrm{CH}_{2} \mathrm{Cl}_{2}\right.$ /petroleum ether). IR: 3250-2650vs (br.), 1463m, 1389m, 1379m, 1325s, 1165s, $1091 m, 1076 m, 942 w, 773 s, 705 m, 643 m .{ }^{1} \mathrm{H}-\mathrm{NMR}\left(\mathrm{CD}_{3} \mathrm{OD}\right): 8.37(s, \mathrm{H}-\mathrm{C}(2)) ; 7.56-$ 7.40 ( $m, 5$ arom. $\mathrm{H}) ; 4.07,3.43\left(2 t, 2 \mathrm{CH}_{2}\right) ; 1.76\left(m, \mathrm{CH}_{2}\right) .{ }^{13} \mathrm{C}-\mathrm{NMR}\left(\mathrm{CD}_{3} \mathrm{OD}\right): 131.5$, 130.8, 130.3 (3d, 5 arom. CH); 128.5, 128.4, $127.4\left(3 s\right.$, arom. $\left.\mathrm{C}_{\mathrm{q}}, \mathrm{C}(4), \mathrm{C}(5)\right) ; 128.0(d$, $\mathrm{C}(2)) ; 58.9$, 44.6, 33.8 (3t, $\left.3 \mathrm{CH}_{2}\right) ; 7.6\left(q\right.$, Me). EI-MS: $232\left(22, M^{+}\right), 214(100), 171$ (52), 117 (76), 104 (29), 55 (29). Anal. calc. for $\mathrm{C}_{13} \mathrm{H}_{16} \mathrm{~N}_{2} \mathrm{O}_{2}$ (232.28): C 67.22, H 6.94, N 12.06; found C 67.37, H 7.05, N 12.01 .

1-(2-Hydroxypropyl)-5-methyl-4-phenyl-1H-imidazole 3-Oxide (11h). Yield $\left.{ }^{3}\right)$ : $211 \mathrm{mg}$ (90\%). Colorless solid. M.p. 143-145 (acetone). IR: 3400-2600vs (br.), $1610 m, 1497 m, 1444 m, 1377 s, 1349 s, 1260 m, 1218 m, 1136 m, 1079 m, 1029 m, 767 s$, 720m, 700s, 678m, 608m. ${ }^{1} \mathrm{H}-\mathrm{NMR}\left(\mathrm{CD}_{3} \mathrm{OD}\right): 8.25(s, \mathrm{H}-\mathrm{C}(2))$; $7.58-7.37$ ( $m, 5$ arom. $\mathrm{H})$; 4.07-4.01 ( $\left.m, \mathrm{CH}_{2}\right)$; 3.87-3.82 ( $\left.m,-\mathrm{CH}(\mathrm{OH})-\right) ; 2.28(s, \mathrm{Me}) ; 1.22(d, J=4.6, \mathrm{Me})$. 
${ }^{13} \mathrm{C}-\mathrm{NMR}\left(\mathrm{CD}_{3} \mathrm{OD}\right): 131.2,129.7,129.5$ (3d, 5 arom. $\left.\mathrm{CH}\right) ; 130.4,128.3,125.7(3 s$, arom. $\left.\mathrm{C}_{\mathrm{q}}, \mathrm{C}(4), \mathrm{C}(5)\right) ; 128.4(d, \mathrm{C}(2)) ; 67.3(d, \mathrm{CH}) ; 53.8\left(t, \mathrm{CH}_{2}\right) ; 20.7,9.5(2 q, 2 \mathrm{Me})$. EI-MS: $232\left(62, M^{+}\right), 216(100), 174$ (64), 159 (72), 130 (40), 103 (58). Anal. calc. for $\mathrm{C}_{13} \mathrm{H}_{16} \mathrm{~N}_{2} \mathrm{O}_{2} \cdot 0.125 \mathrm{H}_{2} \mathrm{O}$ (234.53): C 66.58, H 6.98, N 11.94; found $\mathrm{C} 66.89, \mathrm{H} 6.98, \mathrm{~N}$ 11.82 .

1-(2-Hydroxypropyl)-4,5-diphenyl-1H-imidazole 3-Oxide (11i). Yield $\left.{ }^{3}\right): 274 \mathrm{mg}$ (92\%). Colorless solid. M.p. $182-184^{\circ}\left(\mathrm{CH}_{2} \mathrm{Cl}_{2} /\right.$ petroleum ether). IR: 3350-2550vs (br.), $1486 m, 1445 m, 1398 m, 1353 s, 1265 m, 1187 m, 1138 m, 763 s, 701 s, 656 m .{ }^{1} \mathrm{H}-$ NMR (CD $\left.{ }_{3} \mathrm{OD}\right): 8.48(s, \mathrm{H}-\mathrm{C}(2)) ; 7.45-7.28(m, 10$ arom. $\mathrm{H}) ; 3.99-3.77\left(m, \mathrm{CH}_{2}, \mathrm{CH}\right)$; $1.04(d, J=4.2, \mathrm{Me}) .{ }^{13} \mathrm{C}-\mathrm{NMR}\left(\mathrm{CD}_{3} \mathrm{OD}\right): 132.3,131.2,130.9,130.2,129.7,129.1(6 d$, 10 arom. $\mathrm{CH}) ; 131.0,129.2,128.4,127.8\left(4 s, 2\right.$ arom. $\left.\mathrm{C}_{\mathrm{q}}, \mathrm{C}(4), \mathrm{C}(5)\right) ; 129.3(d, \mathrm{C}(2))$; $66.8(d, \mathrm{CH}) ; 54.2\left(t, \mathrm{CH}_{2}\right) ; 20.7(q, \mathrm{Me})$. EI-MS: $294\left(2, M^{+}\right), 278(11), 105$ (100), 77 (35), 43 (46). Anal. calc. for $\mathrm{C}_{18} \mathrm{H}_{18} \mathrm{~N}_{2} \mathrm{O}_{2} \cdot 0.25 \mathrm{H}_{2} \mathrm{O}$ (298.86): C 72.34, H 6.24, N 9.37; found: C 72.29, H 6.24, N 8.98.

1-((2S)-2-Hydroxypropyl)-4,5-dimethyl-1H-imidazole 3-Oxide (11j). Yield after $\mathrm{CC}\left(\mathrm{R}_{\mathrm{f}}=0.59 ; \mathrm{SiO}_{2}, \mathrm{AcOEt} / \mathrm{MeOH}\right.$ 6:4): $141 \mathrm{mg}(82 \%)$. Colorless solid. M.p. 119-120 (acetone). $[\alpha]_{D}^{17}+42$ (c = 1, MeOH). IR: 3350-2600vs (br.), 1629m, 1446m, 1430m, $1398 s, 1380 m, 1340 s, 1331 s, 1303 m, 1190 m, 1139 s, 1116 m, 1081 m, 864 m, 839 m$, 684m. ${ }^{1} \mathrm{H}-\mathrm{NMR}\left(\mathrm{CDCl}_{3}\right): 7.84(s, \mathrm{H}-\mathrm{C}(2)) ; 4.20-3.68(m, \mathrm{CH}) ; 3.75\left(d, J=2.9, \mathrm{CH}_{2}\right)$; 2.13, $2.09(2 s, 2 \mathrm{Me}) ; 1.21(d, J=6.1, \mathrm{Me}) .{ }^{13} \mathrm{C}-\mathrm{NMR}\left(\mathrm{CD}_{3} \mathrm{OD}\right): 127.4(d, \mathrm{C}(2)) ; 126.4$, $124.1(2 s, \mathrm{C}(4), \mathrm{C}(5)) ; 67.2(d, \mathrm{CH}) ; 53.5\left(t, \mathrm{CH}_{2}\right) ; 20.7,8.7,7.1(3 q, 3 \mathrm{Me})$. CI-MS: $171\left(14,[M+1]^{+}\right), 155(100), 153$ (13). Anal. calc. for $\mathrm{C}_{8} \mathrm{H}_{14} \mathrm{~N}_{2} \mathrm{O}_{2} \cdot 0.125 \mathrm{H}_{2} \mathrm{O}(172.46)$ : C 55.72, H 8.33, N 16.24; found C 55.60, H 8.33, N 16.44.

1-[1-(Hydroxymethyl)propyl]-5-methyl-4-phenyl-1H-imidazole 3-Oxide (11k). Yield $\left.{ }^{3}\right): 212 \mathrm{mg}$ (86\%). Colorless solid. M.p. $171-174^{\circ}\left(\mathrm{CH}_{2} \mathrm{Cl}_{2} /\right.$ petroleum ether). IR: 
3250-2550vs (br.), 1496m, 1443m, 1419m, 1351s, 1327m, 1227s, 1082m, 765s, 700s. ${ }^{1} \mathrm{H}-\mathrm{NMR}\left(\mathrm{CDCl}_{3}\right): 8.38(s, \mathrm{H}-\mathrm{C}(2)) ; 7.59-7.42(m, 5$ arom. $\mathrm{H}) ; 4.28-4.16(m, \mathrm{CH}) ; 3.80$ $\left(d, J=4.0, \mathrm{CH}_{2} \mathrm{OH}\right) ; 2.30(s, \mathrm{Me}) ; 1.94-1.85\left(m, \mathrm{CH}_{2}\right) ; 0.94(t, J=5.5, \mathrm{Me}) .{ }^{13} \mathrm{C}-\mathrm{NMR}$ $\left(\mathrm{CD}_{3} \mathrm{OD}\right): 131.3,129.7,129.5(3 d, 5$ arom. $\mathrm{CH})$; 130.1, 128.2, 126.0 (3s, arom. $\mathrm{C}_{\mathrm{q}}$, $\mathrm{C}(4), \mathrm{C}(5)) ; 126.1(d, \mathrm{C}(2)) ; 64.8,25.4\left(2 t, 2 \mathrm{CH}_{2}\right) ; 62.0(d, \mathrm{CH}) ; 10.7,9.7(2 q, 2 \mathrm{Me})$. EI-MS: $246\left(22, M^{+}\right), 215$ (15), 174 (100), 130 (16), 104 (19), 77(16). Anal. calc. for $\mathrm{C}_{14} \mathrm{H}_{18} \mathrm{~N}_{2} \mathrm{O}_{2}$ (246.31): C 68.27, H 7.37, N 11.37; found C 68.00, 7.38, 11.50 .

1-[1-(Hydroxymethyl)propyl]-4,5-diphenyl-1H-imidazole 3-Oxide (111). Yield $\left.{ }^{3}\right)$ : $219 \mathrm{mg}$ (71\%). Colorless solid. M.p. 199-200 (acetone). IR: 3300-2600vs (br.), $1604 m, 1506 m, 1486 m, 1445 m, 1410 m, 1350 s, 1239 m, 1079 m, 1064 m, 759 s, 696 s$, 655m, 645m. ${ }^{1} \mathrm{H}-\mathrm{NMR}\left(\mathrm{CDCl}_{3}\right): 8.34(s, \mathrm{H}-\mathrm{C}(2)) ; 7.58-7.05$ ( $m, 10$ arom. H); 4.08$3.61(m, \mathrm{CH}) ; 3.67\left(d, J=4.8, \mathrm{CH}_{2} \mathrm{OH}\right) ; 1.91-1.47\left(m, \mathrm{CH}_{2}\right) ; 0.68(t, J=7.2, \mathrm{Me}) .{ }^{13} \mathrm{C}-$ NMR (CD $\left.{ }_{3} \mathrm{OD}\right)$ : 132.7, 131.1, 130.9, 130.1, 129.6, 129.1 (6d, 10 arom. $\left.\mathrm{CH}\right)$; 130.7, 130.4, 128.5, $127.8\left(4 s, 2\right.$ arom. $\left.\mathrm{C}_{\mathrm{q}}, \mathrm{C}(4), \mathrm{C}(5)\right) ; 126.8(d, \mathrm{C}(2)) ; 64.7,25.8\left(2 t, 2 \mathrm{CH}_{2}\right)$; $62.2(d, \mathrm{CH}) ; 10.7(q, \mathrm{Me})$. EI-MS: $308\left(29, M^{+\cdot}\right), 292$ (25), $236(100), 165$ (22), 104 (37).

1-((1S,2S)-2-Hydroxy-1-hydroxymethyl-2-phenylethyl)-5-methyl-4-phenyl-1Himidazole 3-Oxide (11m). Yield after $\mathrm{CC}\left(\mathrm{R}_{\mathrm{f}}=0.7, \mathrm{SiO}_{2}, \mathrm{MeOH}\right): 168 \mathrm{mg}(52 \%)$. Colorless solid. M.p. $177-178^{\circ}\left(\mathrm{CH}_{2} \mathrm{Cl}_{2}\right.$ /petroleum ether). $[\alpha]_{D}^{17}+137$ (c $=0.38$, MeOH). IR: 3450-2450vs (br.), 1625m, 1497m, 1452m, 1412m, 1356s, 1232m, 1093m, 1066m, 1047m, 1029m, 764m, 739m, 699s. ${ }^{1} \mathrm{H}-\mathrm{NMR}\left(\mathrm{CD}_{3} \mathrm{OD}\right): 8.48(s, \mathrm{H}-\mathrm{C}(2))$; 7.467.25 (m, 10 arom. $\mathrm{H}) ; 5.16,5.15(d, \mathrm{CHOH}) ; 4.39-4.36(m, \mathrm{CH}) ; 4.02,4.01\left(d, \mathrm{CH}_{2}\right)$; $1.69(s, \mathrm{Me}) .{ }^{13} \mathrm{C}-\mathrm{NMR}\left(\mathrm{CD}_{3} \mathrm{OD}\right): 131.2,129.7,129.6,129.4,129.1,127.1(6 d, 10$ arom. $\mathrm{CH})$; 142.8, 128.1, 127.2, 126.7 (4s, 2 arom. $\left.\mathrm{C}_{\mathrm{q}}, \mathrm{C}(4), \mathrm{C}(5)\right) ; 127.3(d, \mathrm{C}(2))$; 72.9, $65.9(2 d, 2 \mathrm{CH}) ; 63.2\left(t, \mathrm{CH}_{2}\right) ; 9.1(q, \mathrm{Me}) . \mathrm{EI}-\mathrm{MS}: 324\left(2, M^{+\cdot}\right), 200(100), 104$ 
(21), 77 (22). Anal. calc. for $\mathrm{C}_{19} \mathrm{H}_{20} \mathrm{~N}_{2} \mathrm{O}_{3}$ (324.38): C 70.35, H 6.21, N 8.64; found C 70.51, H 6.25, N 8.74.

5. General Procedure for Synthesis of Acetates 13. To a soln. of $1 \mathrm{mmol}$ of $1 \mathrm{H}$ imidazole $\mathrm{N}$-oxide 11 in abs. $\mathrm{CH}_{2} \mathrm{Cl}_{2}(2 \mathrm{ml})$ in a $\mathrm{H}_{2} \mathrm{O}$ /ice cooling bath, a soln. of freshly distilled $\mathrm{Ac}_{2} \mathrm{O}(0.61 \mathrm{~g}, 6 \mathrm{mmol})$ in abs. $\mathrm{CH}_{2} \mathrm{Cl}_{2}(2 \mathrm{ml})$ was added portion-wise. The mixture was allowed to warm up to r.t., and stirring was continued until $\mathbf{1 1}$ had been consumed (TLC monitoring). Then, the mixture was diluted with $\mathrm{MeOH}$ (5 ml). After stirring for another $30 \mathrm{~min}$, the solvents were evaporated, $\mathrm{H}_{2} \mathrm{O}(5 \mathrm{ml})$ was added, and the white precipitate of the corresponding acetate $\mathbf{1 3}$ was filtered. Analytically pure products were obtained by recrystallization.

\section{2-(2,3-Dihydro-5-methyl-2-oxo-4-phenylimidazol-1-yl)ethyl Acetate (13a).}

Reaction time 2 h. Yield: 185 mg (71\%). Colorless solid. M.p. 135-137 $\left(\mathrm{EtOH} / \mathrm{H}_{2} \mathrm{O}\right)$. IR: 3150-2750s (br., NH), 1745vs (C=O(Ac)), 1672vs (br., $\mathrm{C}=\mathrm{O}), 1456 m, 1429 m$, $1403 m, 1388 m, 1367 m, 1255 s, 1240 s, 1070 m, 769 m, 750 m, 702 m .{ }^{1} \mathrm{H}-\mathrm{NMR}\left(\mathrm{CDCl}_{3}\right)$ : 10.89 (br. $s, \mathrm{NH}) ; 7.44-7.21(m, 5$ arom. $\mathrm{H}) ; 4.30,3.93\left(2 t, 2 \mathrm{CH}_{2}\right) ; 2.25,2.01(2 s, 2$ Me). ${ }^{13} \mathrm{C}-\mathrm{NMR}\left(\mathrm{CDCl}_{3}\right): 170.7(s, \mathrm{C}=\mathrm{O}(\mathrm{Ac})) ; 154.6(s, \mathrm{C}=\mathrm{O}$ (imidazole) $) ; 130.5,118.2$, $115.6\left(3 s\right.$, arom. $\left.\mathrm{C}_{\mathrm{q}}, \mathrm{C}(4), \mathrm{C}(5)\right) ; 128.8,126.7,126.3$ (3d, 5 arom. $\left.\mathrm{CH}\right) ; 62.5,39.7(2 t, 2$ $\left.\mathrm{CH}_{2}\right)$; 20.8, $9.8(2 q, 2 \mathrm{Me})$. CI-MS: $261\left(100,[M+1]^{+}\right)$. Anal. calc. for $\mathrm{C}_{14} \mathrm{H}_{16} \mathrm{~N}_{2} \mathrm{O}_{3}$ (260.30): C 64.60, H 6.20, N 10.76; found: C 64.27, H 5.98, N 10.51.

2-(2,3-Dihydro-2-oxo-4,5-diphenylimidazol-1-yl)ethyl Acetate (13b). Reaction time 2 h. Yield: 303 mg (94\%). Colorless solid. M.p. 187-190 (EtOH). IR: 32002750s (br., NH), 1739s (C=O(Ac)), 1686vs (br., $\mathrm{C}=\mathrm{O}$ ), 1602m, 1506m, 1454m, 1444m, 1432m, 1395m, 1370m, 1234s, 1045m, 768m, 753m, 701m, 667m. ${ }^{1} \mathrm{H}-\mathrm{NMR}\left(\mathrm{CDCl}_{3}\right)$ : 11.69 (br. $s, \mathrm{NH}) ; 7.47-7.11(m, 10$ arom. $\mathrm{H}) ; 4.18,3.88\left(2 t, 2 \mathrm{CH}_{2}\right) ; 1.89(s, \mathrm{Me}) .{ }^{13} \mathrm{C}-$ $\operatorname{NMR}\left(\mathrm{CDCl}_{3}\right)$ : 170.6 ( $\left.s, \mathrm{C}=\mathrm{O}(\mathrm{Ac})\right) ; 154.7$ ( $s, \mathrm{C}=\mathrm{O}$ (imidazole)); 131.2, 129.2, 129.0, 
128.5, 126.8, 125.7 (6d, 10 arom. CH); 129.7, 129.6, 120.7, 119.2 (4s, 2 arom. $\mathrm{C}_{\mathrm{q}}, \mathrm{C}(4)$, $\mathrm{C}(5)) ; 62.1,40.1\left(2 t, 2 \mathrm{CH}_{2}\right) ; 20.8$ (q, Me). CI-MS: $323\left(100,[M+1]^{+}\right), 263$ (5). Anal. calc. for $\mathrm{C}_{19} \mathrm{H}_{18} \mathrm{~N}_{2} \mathrm{O}_{3}$ (322.37): C 70.79, H 5.63, N 8.69; found: C 70.80, H 5.76, N 8.72. 3-(2,3-Dihydro-5-methyl-2-oxo-4-phenylimidazol-1-yl)propyl Acetate (13c). Reaction time 3 h. Yield: 200 mg (73\%). Colorless solid. M.p. 153-154º (EtOH). IR: 3200-2850s (br., $\mathrm{NH}), 1734 v s(\mathrm{C}=\mathrm{O}(\mathrm{Ac})), 1675 v s$ (br., $\mathrm{C}=\mathrm{O}), 1603 m, 1503 w, 1465 m$, 1404m, 1388m, 1364m, 1254s, 1042m, 767m, 747m, 699m. ${ }^{1} \mathrm{H}-\mathrm{NMR}\left(\mathrm{CDCl}_{3}\right): 10.64$ (br. $s, \mathrm{NH}) ; 7.29-7.10$ (m, 5 arom. H); 3.99, $3.64\left(2 t, 2 \mathrm{CH}_{2}\right) ; 2.10,1.90(2 s, 2 \mathrm{Me}) ; 1.88$ $\left(m, \mathrm{CH}_{2}\right) .{ }^{13} \mathrm{C}-\mathrm{NMR}\left(\mathrm{CDCl}_{3}\right): 170.9(s, \mathrm{C}=\mathrm{O}(\mathrm{Ac})) ; 154.0(s, \mathrm{C}=\mathrm{O}($ imidazole $)) ; 130.2$, 118.3, $115.5\left(3 s\right.$, arom. $\left.\mathrm{C}_{\mathrm{q}}, \mathrm{C}(4), \mathrm{C}(5)\right) ; 128.7,126.8,126.3$ (3d, 5 arom. $\left.\mathrm{CH}\right) ; 61.8$, 38.0, $28.5\left(3 t, 3 \mathrm{CH}_{2}\right)$; 20.8, $9.6(2 q, 2 \mathrm{Me})$. EI-MS: $275\left(16,[M+1]^{+}\right), 274\left(100, M^{+\cdot}\right)$, 215 (66), 174 (36), 101 (63), 77 (18). Anal. calc. for $\mathrm{C}_{15} \mathrm{H}_{18} \mathrm{~N}_{2} \mathrm{O}_{3}$ (274.32): C 65.68, $\mathrm{H}$ 6.61, N 10.21; found: C 66.08, H 6.63, N 10.01.

6. Preparation of 1,3-Dihydroimidazole-2-thiones 14. To a soln. of an imidazole $N$-oxide 11 (1 mmol) in $\mathrm{MeOH}(2 \mathrm{ml})$, a soln. of 2,2,4,4-tetramethylcyclobutane-1,3dithione (95 mg, $0.55 \mathrm{mmol})$ in $\mathrm{CHCl}_{3}(2 \mathrm{ml})$ was added drop-wise at $0^{\circ}$, and magnetical stirring was continued for $30 \mathrm{~min}$. Then, the solvents were evaporated i.v., the resulting solid was washed with $\mathrm{Et}_{2} \mathrm{O}$, and the colorless product was filtered and dried i.v. Analytically pure samples were obtained by recrystallization from appropriate solvent.

1,3-Dihydro-1-(2-hydroxyethyl)-4,5-dimethylimidazole-2-thione (14a). Yield: $148 \mathrm{mg}(86 \%)$. Colorless crystals. M.p. 179-180 (MeOH). IR: 3350-2750vs (br., NH), $1659 m, 1506 m, 1444 m, 1402 \mathrm{~s}, 1363 m, 1224 w, 1186 w, 1058 s, 870 w .{ }^{1} \mathrm{H}-\mathrm{NMR}$ ((D)DMSO): 3.92, $3.61\left(2 t, 2 \mathrm{CH}_{2}\right) ; 2.06,1.96(2 s, 2 \mathrm{Me}) .{ }^{13} \mathrm{C}-\mathrm{NMR}\left(\left(\mathrm{D}_{6}\right) \mathrm{DMSO}\right)$ : $168.3(s, \mathrm{C}=\mathrm{S}) ; 131.3,127.8(2 s, \mathrm{C}(4), \mathrm{C}(5)) ; 68.1,55.6\left(2 t, 2 \mathrm{CH}_{2}\right) ; 18.1,18.0(2 q, 2$ 
Me). EI-MS: $172\left(92, M^{+}\right), 128$ (100), 95 (39). Anal. calc. for $\mathrm{C}_{7} \mathrm{H}_{12} \mathrm{~N}_{2} \mathrm{OS}$ (127.25): C 48.81, H 7.02, N 16.26; found: C 49.11, H 7.06, N 15.70.

1,3-Dihydro-1-(3-hydroxypropyl)-5-methyl-4-phenylimidazole-2-thione

(14b).

Yield: $231 \mathrm{mg}$ (93\%). Colorless crystals. M.p. $172-174^{\circ}\left(\mathrm{CH}_{2} \mathrm{Cl}_{2} /\right.$ petroleum ether). IR: 3350-2750vs (br., NH), 1497s, 1459m, 1407s, 1376m, 1282m, 1213m, 1196m, 1174s, 1087m, 1068s, 985m, 933m, 769s, 708m, 699s. ${ }^{1} \mathrm{H}-\mathrm{NMR}\left(\left(\mathrm{D}_{6}\right) \mathrm{DMSO}\right): 12.42$ (br.s, $\mathrm{NH}) ; 7.48-7.31(m, 5$ arom. $\mathrm{H}) ; 4.08,3.46\left(2 t, 2 \mathrm{CH}_{2}\right) ; 2.31(\mathrm{~s}, \mathrm{Me}) ; 1.83\left(m, \mathrm{CH}_{2}\right) .{ }^{13} \mathrm{C}-$ NMR ((D $)$ DMSO): $169.9(s, \mathrm{C}=\mathrm{S}) ; 138.4\left(s\right.$, arom. $\left.\mathrm{C}_{\mathrm{q}}\right) ; 138.3,136.9,136.4(3 d, 5$ arom. $\mathrm{CH}) ; 133.0,132.1(2 s, \mathrm{C}(4), \mathrm{C}(5)) ; 67.4,50.6,40.9\left(3 t, 3 \mathrm{CH}_{2}\right) ; 19.2(q, \mathrm{Me}) . \mathrm{EI}-$ MS: $248\left(41, M^{+}\right), 230$ (59), 215 (100), 204 (27). Anal. calc. for $\mathrm{C}_{13} \mathrm{H}_{16} \mathrm{~N}_{2} \mathrm{OS}$ (248.35): C 62.87, H 6.49, N 11.28; found: C 62.90, H 6.48, N 11.10.

7. Preparation of 1,1'-(Alkane-1,n-diyl)bisimidazole 3,3'-Dioxides 16. A soln. of a diamine $15(1 \mathrm{mmol})$, paraformaldehyde $(75 \mathrm{mg}, 2.5 \mathrm{mmol})$ or acetaldehyde $(110 \mathrm{mg}$, $2.5 \mathrm{mmol})$ and $\mathbf{1 0}(2 \mathrm{mmol})$ in EtOH was heated to reflux for $3 \mathrm{~h}$. Then, the solvent was evaporated i.v. To the resulting oil, acetone $(10 \mathrm{ml})$ was added, the soln. was heated to reflux, and after cooling, the colorless precipitate was collected as highly pure product.

1,1'-(Ethane-1,2-diyl)bis(4,5-dimethylimidazole) 3,3'-Dioxide (16a). Yield: 137 mg (48\%). Colorless solid. M.p. (dec.) 231-236 (EtOH/Et $2 \mathrm{O})$. IR: 3650-2800vs (br.), $1629 m, 1451 m, 1400 s, 1386 s, 1358 m, 1336 s, 1154 m, 834 m, 790 m, 620 m, 603 m .{ }^{1} \mathrm{H}-$ $\operatorname{NMR}\left(\mathrm{CDCl}_{3}\right): 8.15\left(s, \mathrm{H}-\mathrm{C}(2), \mathrm{H}-\mathrm{C}\left(2^{\prime}\right)\right) ; 4.33\left(s, 2 \mathrm{CH}_{2}\right) ; 2.13,1.99(2 s, 4 \mathrm{Me}) .{ }^{13} \mathrm{C}-$ NMR $\left(\mathrm{CDCl}_{3}\right)$ : 127.5, $124.1\left(2 s, \mathrm{C}(4), \mathrm{C}\left(4^{\prime}\right), \mathrm{C}(5), \mathrm{C}\left(5^{\prime}\right)\right) ; 127.1\left(d, \mathrm{C}(2), \mathrm{C}\left(2^{\prime}\right)\right) ; 46.7$ $\left(t, 2 \mathrm{CH}_{2}\right) ; 7.9,7.1(2 q, 4 \mathrm{Me})$. ESI-MS: $273\left(100,[M+\mathrm{Na}]^{+}\right), 251\left(2,[M+1]^{+}\right)$. Anal. calc. for $\mathrm{C}_{12} \mathrm{H}_{18} \mathrm{~N}_{4} \mathrm{O}_{2} \cdot 2 \mathrm{H}_{2} \mathrm{O}$ (286.34): C 50.34, $\mathrm{H}$ 7.74, N 19.57; found C 50.05, $\mathrm{H}$ 8.34, N 19.68 . 


\section{1,1'-(Ethane-1,2-diyl)bis(5-methyl-4-phenylimidazole) 3,3'-Dioxide (16b).}

Yield: $172 \mathrm{mg}$ (42\%). Colorless crystals. M.p. (dec.) 236-240 (MeOH/EtOH). IR: 3550-2700vs (br.), 1679m, 1498m, 1399s, 1361m, 1344m, 1268m, 1228m, 764s, 707m, 697m, 628m, 601m. ${ }^{1} \mathrm{H}-\mathrm{NMR}\left(\mathrm{CD}_{3} \mathrm{OD}\right): 8.68\left(s, \mathrm{H}-\mathrm{C}(2), \mathrm{H}-\mathrm{C}\left(2^{\prime}\right)\right) ; 7.85-7.67(m, 10$ arom. $\mathrm{H}) ; 4.73\left(s, 2 \mathrm{CH}_{2}\right) ; 2.37(s, 2 \mathrm{Me}) .{ }^{13} \mathrm{C}-\mathrm{NMR}\left(\mathrm{CD}_{3} \mathrm{OD}\right): 131.3,127.2,125.4(3 s$, 2 arom. $\left.\mathrm{C}_{\mathrm{q}}, \mathrm{C}(4), \mathrm{C}\left(4^{\prime}\right), \mathrm{C}(5), \mathrm{C}\left(5^{\prime}\right)\right) ; 131.1,130.1,129.6(3 d, 10$ arom. $\mathrm{CH}) ; 127.9$ (d, $\left.\mathrm{C}(2), \mathrm{C}\left(2^{\prime}\right)\right) ; 46.9\left(t, 2 \mathrm{CH}_{2}\right) ; 8.9(q, 2 \mathrm{Me})$. ESI-MS: $397\left(100,[M+\mathrm{Na}]^{+}\right), 375(6$, $\left.[M+1]^{+}\right)$. Anal. calc. for $\mathrm{C}_{22} \mathrm{H}_{22} \mathrm{~N}_{4} \mathrm{O}_{2} \cdot 2 \mathrm{H}_{2} \mathrm{O}$ (410.49): C 64.38, H 6.38, N 13.65; found: C 64.32, H 6.33, N 13.60.

Suitable crystals for the X-ray crystal structure determination were grown from EtOH by slow evaporation of the solvent at r.t.

1,1'-(Ethane-1,2-diyl)bis(2,5-dimethyl-4-phenylimidazole) 3,3'-Dioxide (16c). Yield: $153 \mathrm{mg}$ (35\%). Colorless solid. M.p. (dec.) 234-237 (acetone). IR: 3450-2850vs (br.), 1626m, 1516m, 1490m, 1462m, 1445m, 1414m, 1382m, 1348s, 1318m, 1274s, 1224m, 769s, 703s, 656m, 597s. ${ }^{1} \mathrm{H}-\mathrm{NMR}\left(\mathrm{CD}_{3} \mathrm{OD}\right): 7.52-7.38$ (m, 10 arom. H); 4.35 $\left(s, 2 \mathrm{CH}_{2}\right) ; 2.48,2.05(2 s, 4 \mathrm{Me}) .{ }^{13} \mathrm{C}-\mathrm{NMR}\left(\mathrm{CD}_{3} \mathrm{OD}\right): 134.5,130.0,126.7,121.9(4 s, 2$ arom. $\left.\mathrm{C}_{\mathrm{q}}, \mathrm{C}(2), \mathrm{C}\left(2^{\prime}\right), \mathrm{C}(4), \mathrm{C}\left(4^{\prime}\right), \mathrm{C}(5), \mathrm{C}\left(5^{\prime}\right)\right)$; 130.2, 129.4, 128.9 (3d, 6 arom. $\left.\mathrm{CH}\right)$; $44.2\left(t, \mathrm{CH}_{2}\right) ; 8.7,7.9(2 q, 4 \mathrm{Me})$. EI-MS: 402 (22, $\left.M^{+}\right), 386$ (36), 355 (100), 213 (33), 172 (56), 103 (52). Anal. calc. for $\mathrm{C}_{24} \mathrm{H}_{26} \mathrm{~N}_{4} \mathrm{O}_{2} \cdot 2 \mathrm{H}_{2} \mathrm{O}$ (438.54): C 65.73, H 6.90, N 12.78; found: C 66.23, H 6.89, N 12.53 .

1,1'-(Propane-1,3-diyl)bis(4,5-dimethylimidazole) 3,3'-Dioxide (16d). Yield: 33 mg (11\%). Colorless solid. M.p. (dec.) 191-196º IR: 3550-2800vs (br.), 1686m, $1626 m, 1472 m, 1403 m, 1380 s, 1345 s, 1200 m, 1149 m, 1077 m, 695 m, 618 m, 582 m .{ }^{1} \mathrm{H}-$ NMR (CD $\left.{ }_{3} \mathrm{OD}\right): 8.22\left(s, \mathrm{H}-\mathrm{C}(2), \mathrm{H}-\mathrm{C}\left(2^{\prime}\right)\right) ; 4.02\left(t, 2 \mathrm{CH}_{2}\right) ; 2.23\left(m, \mathrm{CH}_{2}\right) ; 2.20,2.14$ $(2 s, 4 \mathrm{Me}) .{ }^{13} \mathrm{C}-\mathrm{NMR}\left(\mathrm{CD}_{3} \mathrm{OD}\right): 127.2,126.8$ (2s, $\left.\mathrm{C}(4), \mathrm{C}\left(4^{\prime}\right), \mathrm{C}(5), \mathrm{C}\left(5^{\prime}\right)\right) ; 123.7$ (d, 
$\left.\mathrm{C}(2), \mathrm{C}\left(2^{\prime}\right)\right)$; 43.8, $31.7\left(2 t, 3 \mathrm{CH}_{2}\right) ; 8.3,7.0(2 q, 4 \mathrm{Me})$. EI-MS: $264\left(3, M^{+}\right), 232(26)$, 137 (51), 123 (100), 110 (93), 96 (57). Anal. calc. for $\mathrm{C}_{13} \mathrm{H}_{20} \mathrm{~N}_{4} \mathrm{O}_{2} \cdot 2 \mathrm{H}_{2} \mathrm{O}$ (300.37): C 51.99, H 8.05, N 18.65; found: C 51.70, H 8.12, N 17.75.

1,1'-(Butane-1,4-diyl)bis(4,5-dimethylimidazole) 3,3'-Dioxide (16e). Yield: 156 mg (47\%). Colorless solid. M.p. (dec.) 151-155 (EtOH). IR (KBr): 3550-2850vs (br.), $1625 m, 1401 m, 1377 m, 1341 s, 1228 m, 1160 m, 1142 m, 682 m, 625 m .{ }^{1} \mathrm{H}-\mathrm{NMR}$ $\left(\mathrm{CD}_{3} \mathrm{OD}\right): 8.19\left(s, \mathrm{H}-\mathrm{C}(2), \mathrm{H}-\mathrm{C}\left(2^{\prime}\right)\right) ; 4.00\left(t, 2 \mathrm{CH}_{2}\right) ; 2.20,2.14(2 s, 4 \mathrm{Me}) ; 1.81-1.75$ $\left(m, 2 \mathrm{CH}_{2}\right) .{ }^{13} \mathrm{C}-\mathrm{NMR}\left(\mathrm{CD}_{3} \mathrm{OD}\right): 127.0,123.6\left(2 s, \mathrm{C}(4), \mathrm{C}\left(4^{\prime}\right), \mathrm{C}(5), \mathrm{C}\left(5^{\prime}\right)\right) ; 126.7$ (d, $\left.\mathrm{C}(2), \mathrm{C}\left(2^{\prime}\right)\right)$; 46.2, $28.1\left(2 t, 4 \mathrm{CH}_{2}\right) ; 8.4,7.1(2 q, 4 \mathrm{Me})$. ESI-MS: $279\left(100,[M+1]^{+}\right)$. Anal. calc. for $\mathrm{C}_{14} \mathrm{H}_{22} \mathrm{~N}_{4} \mathrm{O}_{2} \cdot 3 \mathrm{H}_{2} \mathrm{O}$ (332.42): C 50.59, H 8.49, N 16.86; found: C 50.40, H 8.18, N 16.50.

1,1'-(Hexane-1,6-diyl)bis(4,5-dimethylimidazole) 3,3'-Dioxide (16f). Yield: 162 mg (43\%). Colorless solid. M.p. (dec.) 101-104 (EtOH/Et $2 \mathrm{O})$. IR: 3500-2950vs (br.), $1627 m, 1481 m, 1413 m, 1381 m, 1336 m, 1193 m, 1144 m, 1088 m, 738 m, 589 m .{ }^{1} \mathrm{H}-\mathrm{NMR}$ $\left(\mathrm{CDCl}_{3}\right): 7.89\left(s, \mathrm{H}-\mathrm{C}(2), \mathrm{H}-\mathrm{C}\left(2^{\prime}\right)\right) ; 3.84\left(t, 2 \mathrm{CH}_{2}\right) ; 2.15$ (br. $\left.s, 4 \mathrm{Me}\right) ; 1.82-1.66$, 1.40-1.29 (2m, $\left.4 \mathrm{CH}_{2}\right) .{ }^{13} \mathrm{C}-\mathrm{NMR}\left(\mathrm{CDCl}_{3}\right): 127.1\left(d, \mathrm{C}(2), \mathrm{C}\left(2^{\prime}\right)\right) ; 126.0,121.2(2 s$, $\left.\mathrm{C}(4), \mathrm{C}\left(4^{\prime}\right), \mathrm{C}(5), \mathrm{C}\left(5^{\prime}\right)\right)$; 45.4, 29.8, $25.6\left(3 t, 6 \mathrm{CH}_{2}\right)$; 8.2, 6.3 (2q, $\left.4 \mathrm{Me}\right)$. EI-MS: 306 (21, $\left.M^{+\cdot}\right), 290$ (47), 273 (51), 179 (59), 165 (100), 151 (66), 137 (56), 110 (70). Anal. calc. for $\mathrm{C}_{16} \mathrm{H}_{26} \mathrm{~N}_{4} \mathrm{O}_{2} \cdot 4 \mathrm{H}_{2} \mathrm{O}$ (378.49): C 50.78, H 9.06, N 14.80; found: C 50.39, $\mathrm{H}$ $9.15, \mathrm{~N} 14.40$.

\section{1,1'-(Hexane-1,6-diyl)bis(5-methyl-4-phenylimidazole) 3,3'-Dioxide (16g).} Yield: $247 \mathrm{mg}$ (53\%). Colorless solid. M.p. (dec.) 188-192 (EtOH/Et $\left.{ }_{2} \mathrm{O}\right)$. IR: 3600 $2750 v s$ (br.), 1646m, 1497m, 1470m, 1429m, 1390s, 1365s, 1347s, 1250m, 1216m, 835m, 762s, 699s, 598s. ${ }^{1} \mathrm{H}-\mathrm{NMR}\left(\mathrm{CD}_{3} \mathrm{OD}\right): 8.34\left(s, \mathrm{H}-\mathrm{C}(2), \mathrm{H}-\mathrm{C}\left(2^{\prime}\right)\right)$; 7.60-7.37 (m, 10 arom. $\mathrm{H}) ; 4.04\left(t, 2 \mathrm{CH}_{2}\right) ; 2.28(s, 2 \mathrm{Me}) ; 1.84\left(t, 2 \mathrm{CH}_{2}\right) ; 1.49-1.44\left(m, 2 \mathrm{CH}_{2}\right) .{ }^{13} \mathrm{C}-$ 
NMR (CD $\left.{ }_{3} \mathrm{OD}\right)$ : 131.1, 129.7, 129.4, 127.7 (4d, 10 arom. $\left.\mathrm{CH}, \mathrm{C}(2), \mathrm{C}\left(2^{\prime}\right)\right)$; 130.6, 128.1, $125.0\left(3 s, 2\right.$ arom. $\left.\mathrm{C}_{\mathrm{q}}, \mathrm{C}(4), \mathrm{C}\left(4^{\prime}\right), \mathrm{C}(5), \mathrm{C}\left(5^{\prime}\right)\right)$; 47.0, 31.0, 26.9 (3t, $\left.6 \mathrm{CH}_{2}\right) ; 9.3$ (q, $2 \mathrm{Me})$. ESI-MS: $431\left(17,[M+1]^{+}\right)$. Anal. calc. for $\mathrm{C}_{26} \mathrm{H}_{30} \mathrm{~N}_{4} \mathrm{O}_{2} \cdot 2 \mathrm{H}_{2} \mathrm{O}$ (466.59): C 66.93, H 7.35, N 12.01; found C 67.31, H 7.48, N 12.04.

8. Synthesis of N-[2-(5-methyl-3-oxy-4-phenylimidazol-1-yl)alkyl]acetamides $\mathbf{1 8 .}$ Step 1: AcOEt $(0.88 \mathrm{~g}, 10 \mathrm{mmol})$ was added to a fourfold excess of a diamine $\mathbf{1 5}$ in $\mathrm{MeOH}(25 \mathrm{ml})$, and the resulting soln. was allowed to stand at r.t. for 4 d. Then, solvent, by-product $\mathrm{EtOH}$, excess AcOEt and diamine were removed i.v. to give the corresponding $N$-acetylalkyldiamine $\mathbf{1 7}$ as a colorless oily product.

Step 2: To a soln. of $\mathbf{1 7}$ in $\mathrm{MeOH}(5 \mathrm{ml})$, paraformaldehyde $(0.156 \mathrm{~g}, 5.2 \mathrm{mmol})$ was added at r.t. and the mixture was stirred for $24 \mathrm{~h}$. Then, the soln. was filtered and the solvent removed i.v. to give the corresponding imine as a yellow oil in almost quantitative yield. The crude products were used in the next step without purification.

Step 3: A soln. of the corresponding monoxime dione $\mathbf{1 0}(1 \mathrm{mmol})$ and $1.2 \mathrm{mmol}$ of the diamine derivative obtained in Step 2 in EtOH $(10 \mathrm{ml})$ was refluxed for $3 \mathrm{~h}$. After evaporation of the solvent, the resulting oil was treated with acetone, warmed and cooled again. The white precipitate of $\mathbf{1 8}$ was collected and recrystallized from an appropriate solvent.

N-[2-(5-Methyl-3-oxy-4-phenylimidazol-1-yl)ethyl]acetamide (18a). Yield: 161 mg (58\%). Colorless solid. M.p. 174-175 (acetone). IR: 3500-2850vs (br.), 1647vs $(\mathrm{C}=\mathrm{O}), 1560 m, 1444 m, 1397 m, 1382 m, 1348 m, 1308 m, 1284 m, 1258 m, 1213 m, 769 m$, 700m, 601m. ${ }^{1} \mathrm{H}-\mathrm{NMR}\left(\mathrm{CDCl}_{3}\right): 8.66$ (br. $\left.t, \mathrm{NH}\right) ; 8.35\left(s, \mathrm{H}-\mathrm{C}\left(2^{\prime}\right)\right) ; 7.56-7.29(m, 5$ arom. $\mathrm{H}) ; 3.89\left(t, \mathrm{CH}_{2}\right) ; 3.31-3.24\left(m, \mathrm{CH}_{2}\right) ; 2.19,1.75(2 s, 2 \mathrm{Me}) .{ }^{13} \mathrm{C}-\mathrm{NMR}\left(\mathrm{CDCl}_{3}\right)$ : $171.7(s, \mathrm{C}=\mathrm{O})$; 129.7, 128.6, 128.4, 125.9 (4d, 5 arom. $\left.\mathrm{CH}, \mathrm{H}-\mathrm{C}\left(2^{\prime}\right)\right)$; 129.3, 127.2, $123.2\left(3 s\right.$, arom. $\left.\mathrm{C}_{\mathrm{q}}, \mathrm{C}\left(4^{\prime}\right), \mathrm{C}\left(5^{\prime}\right)\right)$; 44.9, $39.0\left(2 t, 2 \mathrm{CH}_{2}\right)$; 22.5, 9.3 (2q, $\left.2 \mathrm{Me}\right)$. CI-MS: 
$260\left(56,[M+1]^{+}\right), 244(100), 232(9), 230$ (10), 159 (6). Anal. calc. for $\mathrm{C}_{14} \mathrm{H}_{17} \mathrm{~N}_{3} \mathrm{O}_{2}$. $\mathrm{H}_{2} \mathrm{O}$ (277.33): C 60.63, H 6.91, N 15.15, found: C 60.54, H 6.39, N 15.06.

N-[3-(4,5-Dimethyl-3-oxyimidazol-1-yl)propyl]acetamide (18b). Yield: $165 \mathrm{mg}$ (78\%). Colorless needles. M.p. $170-171^{\circ}\left(\mathrm{CH}_{2} \mathrm{Cl}_{2} /\right.$ petroleum ether). IR: 3350-2850vs (br.), 1667vs (C=O), 1559m, 1446m, 1400m, 1383m, 1371m, 1338m, 1293m, 609m. ${ }^{1} \mathrm{H}-$ $\operatorname{NMR}\left(\mathrm{CDCl}_{3}\right): 8.23(s, \mathrm{H}-\mathrm{C}(2)) ; 3.98,3.20\left(2 t, 2 \mathrm{CH}_{2}\right) ; 2.20,2.14,1.94(3 s, 3 \mathrm{Me})$; $1.93\left(m, \mathrm{CH}_{2}\right) \cdot{ }^{13} \mathrm{C}-\mathrm{NMR}\left(\mathrm{CDCl}_{3}\right): 173.4(s, \mathrm{C}=\mathrm{O}) ; 127.0,123.6\left(2 s, \mathrm{C}\left(4^{\prime}\right), \mathrm{C}\left(5^{\prime}\right)\right)$; $126.9\left(d, \mathrm{C}\left(2^{\prime}\right)\right)$; 44.5, 37.3, $31.1\left(3 t, 3 \mathrm{CH}_{2}\right) ; 22.6,8.3,7.1(3 q, 3 \mathrm{Me})$. EI-MS: 211 (38, $M^{+}$), 110 (80), 100 (100), 97 (28), 72 (27). Anal. calc. for $\mathrm{C}_{10} \mathrm{H}_{17} \mathrm{~N}_{3} \mathrm{O}_{2} \cdot 0.25 \mathrm{H}_{2} \mathrm{O}$ (215.77): C 55.67, H 8.18, N 19.47, found: C 55.76, H 8.27, N 18.59.

9. Synthesis of 1,1'-(Ethane-1,2-diyl)bis(2,3-dihydro-4,5-dimethylimidazol-2one) (19). To a soln. of $16 \mathrm{a}(286 \mathrm{mg}, 1 \mathrm{mmol})$ in $\mathrm{CHCl}_{3}(10 \mathrm{ml})$, a soln. of $\mathrm{Ac}_{2} \mathrm{O}(2$ $\mathrm{ml})$ diluted $\mathrm{CHCl}_{3}(2 \mathrm{ml})$ was added, and the mixture was heated to refluxed for $3 \mathrm{~h}$. After cooling, $\mathrm{MeOH}(5 \mathrm{ml})$ was added carefully, and stirring was continued for $30 \mathrm{~min}$. Then, the solvent was removed, $\mathrm{H}_{2} \mathrm{O}(5 \mathrm{ml})$ was added, and the colorless $\mathbf{1 9}$, containing small amounts of $N$-acetylated derivatives, was filtered and purified by crystallization from aq. MeOH. Yield of 19: $140 \mathrm{mg}$ (43\%). Colorless solid. M.p. 218-223 ${ }^{\circ}$ $\left(\mathrm{MeOH} / \mathrm{H}_{2} \mathrm{O}\right)$. IR: 3450-2750vs (br.), 1671vs (C=O), 1460m, 1407s, 1373m, 1315w, $1114 w, 747 m, 627 m, 558 m .{ }^{1} \mathrm{H}-\mathrm{NMR}\left(\mathrm{CDCl}_{3}\right): 3.78\left(s, 2 \mathrm{CH}_{2}\right) ; 1.92,1.75$ (2 br. $s, 4$ Me). ${ }^{13} \mathrm{C}-\mathrm{NMR}\left(\mathrm{CDCl}_{3}\right): 155.2(s, \mathrm{C}=\mathrm{O}) ; 115.9,113.7\left(2 s, \mathrm{C}(4), \mathrm{C}\left(4^{\prime}\right), \mathrm{C}(5), \mathrm{C}\left(5^{\prime}\right)\right)$; $41.1\left(t, 2 \mathrm{CH}_{2}\right) ; 9.0,7.7(2 q, 4 \mathrm{Me})$. CI-MS: $251\left(100,[M+1]^{+}\right), 138$ (11). Anal. calc. for $\mathrm{C}_{12} \mathrm{H}_{18} \mathrm{~N}_{4} \mathrm{O}_{2} \cdot 4 \mathrm{H}_{2} \mathrm{O}$ (322.38): $\mathrm{C} 44.71, \mathrm{H} 8.13, \mathrm{~N} 17.38$; found: $\mathrm{C} 44.69, \mathrm{H} 7.94, \mathrm{~N}$ 17.04.

10. General Procedure for the Synthesis of N,N'-Diacetyl-bis(imidazolones) 20: A soln. of an $\mathrm{N}$-oxide $16(1 \mathrm{mmol})$ in freshly distilled $\mathrm{Ac}_{2} \mathrm{O}(2 \mathrm{ml})$ was heated to reflux 
for $2 \mathrm{~h}$. The mixture was cooled and excess $\mathrm{MeOH}(10 \mathrm{ml})$ was added. After evaporation of the solvents i.v., $\mathrm{H}_{2} \mathrm{O}(10 \mathrm{ml})$ was added, and the crude product was filtered and recrystallized.

\section{1,1'-(Ethane-1,2-diyl)bis(3-acetyl-2,3-dihydro-4,5-dimethylimidazol-2-one)}

(20a). Yield: $140 \mathrm{mg}$ (42\%). Colorless solid. M.p. (dec.) 232-238 (MeCN). IR: 1717vs $(\mathrm{C}=\mathrm{O}(\mathrm{Ac})), 1675 s(\mathrm{C}=\mathrm{O}), 1449 m, 1403 s, 1386 s, 1370 s, 1316 s, 1178 m, 1106 m, 750 m$, 579m, 560m. ${ }^{1} \mathrm{H}-\mathrm{NMR}\left(\mathrm{CDCl}_{3}\right): 3.78\left(s, 2 \mathrm{CH}_{2}\right) ; 2.63(s, 2 \mathrm{MeCO}) ; 2.34,1.92(2$ br. $s, 4$ Me). $\left.{ }^{13} \mathrm{C}-\mathrm{NMR}\left(\mathrm{CDCl}_{3}\right): 170.7(s, 2 \mathrm{MeC}=\mathrm{O})\right) ; 152.5(s, 2 \mathrm{C}=\mathrm{O}) ; 117.4,114.0(2 s$, $\left.\left.\mathrm{C}(4), \mathrm{C}\left(4^{\prime}\right), \mathrm{C}(5), \mathrm{C}^{\prime} 5^{\prime}\right)\right) ; 39.4\left(t, 2 \mathrm{CH}_{2}\right) ; 26.0$ ( $\left.q, 2 \mathrm{MeCO}\right) ; 11.8,7.8$ (2q, $\left.4 \mathrm{Me}\right) . \mathrm{CI}-$ MS: 336 (26), $335\left(100,[M+1]^{+}\right), 293$ (7). Anal. calc. for $\mathrm{C}_{16} \mathrm{H}_{22} \mathrm{~N}_{4} \mathrm{O}_{4}$ (334.38): C 57.47, H 6.63, N 16.76; found: C 57.21, H 6.86, N 16.36.

\section{1,1'-(Butane-1,4-diyl)bis(3-acetyl-2,3-dihydro-4,5-dimethylimidazol-2-one)}

(20b). Yield: 76 mg (21\%). Colorless solid. M.p. $188-189^{\circ}\left(\mathrm{MeOH} / \mathrm{H}_{2} \mathrm{O}\right)$. IR: $1709 v \mathrm{~s}$ $(\mathrm{C}=\mathrm{O}(\mathrm{Ac})), 1673 s(\mathrm{C}=\mathrm{O}), 1457 m, 1438 m, 1386 s, 1370 s, 1317 s, 1173 w, 1106 w, 967 w$, $749 w, 678 m, 599 m .{ }^{1} \mathrm{H}-\mathrm{NMR}\left(\mathrm{CDCl}_{3}\right): 3.60\left(t, 2 \mathrm{CH}_{2}\right) ; 2.63(s, 2 \mathrm{MeCO}) ; 2.24,1.97$ (2 br. $\left.s, 4 \mathrm{Me}) ; 1.68-1.65\left(m, 2 \mathrm{CH}_{2}\right) .{ }^{13} \mathrm{C}-\mathrm{NMR}\left(\mathrm{CDCl}_{3}\right): 170.9(s, 2 \mathrm{MeC}=\mathrm{O})\right) ; 152.5(s$, $2 \mathrm{C}=\mathrm{O}) ; 117.4,113.8\left(2 s, \mathrm{C}(4), \mathrm{C}\left(4^{\prime}\right), \mathrm{C}(5), \mathrm{C}\left(5^{\prime}\right)\right) ; 40.5,26.7\left(2 t, 4 \mathrm{CH}_{2}\right) ; 26.1(q, 2$ MeCO); 11.8, 8.2 (2q, 4 Me). CI-MS: 364 (23), $363\left(100,[M+1]^{+}\right), 321$ (10). Anal. calc. for $\mathrm{C}_{18} \mathrm{H}_{26} \mathrm{~N}_{4} \mathrm{O}_{4}$ (362.43): C 59.65, H 7.23, N 15.46; found: C 59.29, H 7.17, N 15.28.

\section{1,1'-(Hexane-1,6-diyl)bis(3-acetyl-2,3-dihydro-4,5-dimethylimidazol-2-one)}

(20c). Yield: $101 \mathrm{mg}$ (25\%). Colorless solid. M.p. (dec.) 167-170 (MeOH). IR: 1710vs $(\mathrm{C}=\mathrm{O}(\mathrm{Ac})), 1674 m(\mathrm{C}=\mathrm{O}), 1448 w, 1407 m, 1390 m, 1368 s, 1312 s, 1166 w, 1106 w$, $1051 w, 969 w, 908 w, 751 m, 676 w, 607 w, 590 m .{ }^{1} \mathrm{H}-\mathrm{NMR}\left(\mathrm{CDCl}_{3}\right): 3.54\left(t, 2 \mathrm{CH}_{2}\right) ; 2.63$ $(s, 2 \mathrm{MeCO}) ; 2.24,1.96$ (2 br. $s, 4 \mathrm{Me}) ; 1.63-1.59,1.40-1.35\left(2 m, 4 \mathrm{CH}_{2}\right) .{ }^{13} \mathrm{C}-\mathrm{NMR}$ $\left(\mathrm{CDCl}_{3}\right): 171.0(s, 2 \mathrm{MeC}=\mathrm{O}) ; 152.4(s, 2 \mathrm{C}=\mathrm{O}) ; 117.5,113.6$ (2s, C(4), C(4'), C(5), 
$\left.\mathrm{C}\left(5^{\prime}\right)\right) ;$ 40.5, 26.7, $26.4\left(3 t, 6 \mathrm{CH}_{2}\right) ; 26.1$ (q, $\left.2 \mathrm{MeCO}\right) ; 11.8,8.2$ (2q, 4 Me). CI-MS: 392 (25), $391\left(100,[M+1]^{+}\right), 349$ (6), 306 (4). Anal. calc. for $\mathrm{C}_{20} \mathrm{H}_{30} \mathrm{~N}_{4} \mathrm{O}_{4} \cdot 0.5 \mathrm{H}_{2} \mathrm{O}$ (399.50): C 60.13, H 7.82, N 14.02; found: C 60.46, H 7.17, N 13.88.

11. Synthesis of 1,1'-(Butane-1,4-diyl)bis(3-acetyl-2,3-dihydro-4,5dimethylimidazol-2-thione) (21). To a soln. of 16e (332 $\mathrm{mg}$ trihydrate, $1 \mathrm{mmol})$, a soln. of 2,2,4,4-tetramethylcyclobutane-1,3-dithione (95 mg, $0.55 \mathrm{mmol})$ in $\mathrm{CHCl}_{3}(2 \mathrm{ml})$ was added drop-wise, and the mixture was stirred overnight at r.t. The precipitate was filtered, washed with cold $\mathrm{EtOH}$ and $\mathrm{Et}_{2} \mathrm{O}$, and the obtained solid was analyzed without

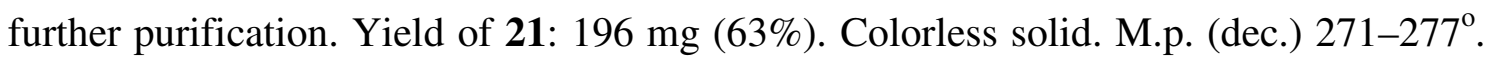
IR: 3450-2700vs (br.), 1659m, 1495s, 1441m, 1406m, 1369m, 1319m, 1250m, 1166m, 780m, 684m. ${ }^{1} \mathrm{H}-\mathrm{NMR}\left(\left(\mathrm{D}_{6}\right) \mathrm{DMSO}\right): 11.81$ (br. $\left.s, 2 \mathrm{NH}\right) ; 3.90\left(t, 2 \mathrm{CH}_{2}\right) ; 2.04,1.95$ (2s, $4 \mathrm{Me}) ; 1.68-1.51\left(m, 2 \mathrm{CH}_{2}\right) .{ }^{13} \mathrm{C}-\mathrm{NMR}\left(\left(\mathrm{D}_{6}\right) \mathrm{DMSO}\right): 158.8(s, \mathrm{C}=\mathrm{S}) ; 120.5,118.7(2 s$, $\left.\mathrm{C}(4), \mathrm{C}\left(4^{\prime}\right), \mathrm{C}(5), \mathrm{C}\left(5^{\prime}\right)\right)$; 43.0, 25.5 (2t, $\left.4 \mathrm{CH}_{2}\right)$; 8.6, 8.4 (2q, 4 Me). EI-MS: 310 (100, $M^{+}$), 183 (84), 149 (54), 128 (32). Anal. calc. for $\mathrm{C}_{14} \mathrm{H}_{22} \mathrm{~N}_{4} \mathrm{~S}_{2}$ (310.49): C 54.16, H 7.14, N 18.04; found C 53.92, H 6.91, N 17.82.

12. Synthesis of 1,1'-(Butane-1,4-diyl)bis(3-acetyl-2,3-dihydro-4,5dimethylimidazole) (22) by deoxygenation of 16f. To a soln. of $16 \mathbf{f}$ (378 $\mathrm{mg}$ tetrahydrate, $1 \mathrm{mmol})$ in EtOH ( $2 \mathrm{ml})$, a suspension of freshly prepared Raney-Nickel in EtOH was added in small portions. The progress of the reaction was monitored by means of TLC (MeOH/AcOEt 1:3). When the $\mathbf{1 6 f}$ was completely reduced, the mixture was filtered and the filtrate was concentrated i.v. The crude product was purified by crystallization from EtOH to give 22. Yield: $302 \mathrm{mg}$ (76\%). Colorless crystals. M.p. 163-164º IR: 3120-2850vs (br.), 1602s, 1510s, 1495m, 1445m, 1371m, 1277m, 1261s, 944m, 850m, 769s, 696vs, 636m. ${ }^{1} \mathrm{H}-\mathrm{NMR}\left(\mathrm{CDCl}_{3}\right):$ 7.67-7.21 (m, 10 arom. H); 7.48 (s, $\left.\mathrm{H}-\mathrm{C}(2), \mathrm{H}-\mathrm{C}\left(2^{\prime}\right)\right) ; 3.87\left(t, 2 \mathrm{CH}_{2}\right) ; 2.38(s, 2 \mathrm{Me}) ; 1.81-1.73,1.43-1.35\left(2 m, 4 \mathrm{CH}_{2}\right)$. 
${ }^{13} \mathrm{C}-\mathrm{NMR}\left(\mathrm{CDCl}_{3}\right): 138.1,135.3,123.0$ (3s, 2 arom. $\left.\mathrm{C}_{\mathrm{q}}, \mathrm{C}(4), \mathrm{C}\left(4^{\prime}\right), \mathrm{C}(5), \mathrm{C}\left(5^{\prime}\right)\right)$; 135.9, 128.4, 127.0, 126.2 (4d, 10 arom. $\left.\mathrm{CH}, \mathrm{C}(2), \mathrm{C}\left(2^{\prime}\right)\right)$; 44.9, 30.6, $26.3\left(3 t, 6 \mathrm{CH}_{2}\right)$; 9.9 (q, 2 Me). ESI-MS: $399\left(100,[M+1]^{+}\right)$. Anal. calc. for $\mathrm{C}_{26} \mathrm{H}_{30} \mathrm{~N}_{4}$ (398.56): C 78.36, H 7.59, N 14.06; found: C 77.92, H 6.90, N 13.79.

13. X-Ray Crystal-Structure Determination of 11a and 16b (Table and Figs. 1$2)^{4}$ ). All measurements were performed on a Nonius KappaCCD diffractometer [27] using graphite-monochromated $\operatorname{MoK}_{\alpha}$ radiation $\left(\begin{array}{lll}\lambda & 0.71073 & \AA\end{array}\right)$ and an Oxford Cryosystems Cryostream 700 cooler. The data collection and refinement parameters are given in the Table, and views of the molecules are shown in Figs. 1 and 2. Data reduction was performed with HKL Denzo and Scalepack [28]. The intensities were corrected for Lorentz and polarization effects but not for absorption. Equivalent reflections were merged. The structures were solved by direct methods using SIR92 [29], which revealed the positions of all non-H-atoms. The asymmetric unit of 11a contains one zwitterionic molecule and half of a $\mathrm{H}_{2} \mathrm{O}$ molecule, which sits on a $\mathrm{C}_{2}$-axis, while that of $\mathbf{1 6 b}$ contains one half of the heterocyclic molecule, which sits across a $C_{2^{-}}$ axis, plus one $\mathrm{H}_{2} \mathrm{O}$ molecule in a general position. The non- $\mathrm{H}$-atoms of $\mathbf{1 1 a}$ and $\mathbf{1 6 b}$ were refined anisotropically. The $\mathrm{H}$-atoms of the $\mathrm{OH}$ group and the $\mathrm{H}_{2} \mathrm{O}$ molecule of 11a and the $\mathrm{H}$-atoms of the $\mathrm{H}_{2} \mathrm{O}$ molecule of $\mathbf{1 6} \mathbf{b}$ were placed in the positions indicated by a difference electron density map and their positions were allowed to refine with individual isotropic displacement parameters. All remaining $\mathrm{H}$-atoms were placed in geometrically calculated positions and refined using a riding model where each $\mathrm{H}$-atom

4) CCDC-646512-646513 contain the supplementary crystallographic data for this paper. These data can be obtained free of charge from the Cambridge Crystallographic Data Centre, via www.ccdc.cam.ac.uk/data_request/cif. 
was assigned a fixed isotropic displacement parameter with a value equal to $1.2 U_{\text {eq }}$ of its parent C-atom (1.5 $U_{\text {eq }}$ for the Me groups). The refinement of each structure was carried out on $F^{2}$ using full-matrix least-squares procedures, which minimized the function $\Sigma w\left(F_{\mathrm{o}}^{2}-F_{\mathrm{c}}{ }^{2}\right)^{2}$. In the case of 11a, a correction for secondary extinction was applied, and one reflection, whose intensities was considered to be an extreme outlier, was omitted from the final refinement. Neutral atom scattering factors for non-H-atoms were taken from [30a], and the scattering factors for H-atoms were taken from [31]. Anomalous dispersion effects were included in $F_{\mathrm{c}}$ [32]; the values for $f^{\prime}$ and $f^{\prime \prime}$ were those of [30b]. The values of the mass attenuation coefficients are those of [30c]. All calculations were performed using the SHELXL97 [33] program. 


\section{REFERENCES}

[1] J. Zhong, Nat. Prod. Rep. 2005, 22, 196; T. D. Heigtman, A. T. Vasella, Angew. Chem., Int. Ed. 1999, 38, 750.

[2] G. Aguirre, M. Boiani, H. Cerecetto, A. Gerpe, M. Gonzales, Y. Fernandez Sainz, A. Denicola, C. Ochoa de Ochariz, J. J. Nogal, D. Montero, J. A. Escarion, Arch. Pharm. Pharm. Med. Chem. 2004, 337, 259.

[3] J. F. Callahan, J. L. Burgess, J. A. Fronwald, L. M. Gaster, J. D. Harling, F. P. Harrington, J. Heer, C. Kwon, R. Lehr, A. Mathur, B. A. Olson, J. Weinstock, N. J. Laping, J. Med. Chem. 2002,45, 999.

[4] I. Kawasaki, N. Sakaguchi, A. Khadeer, M. Yamashita, S. Ohta, Tetrahedron, 2006, 62, 10182.

[5] S. Laufer, G. Wagner, D. Kotschenreuther, Angew. Chem., Int. Ed. 2002, 41, 2290.

[6] M. Shi, H. Qian, Tetrahedron 2005, 61, 4949.

[7] P. Wasserscheid, T. Welton, Ionic Liquids in Synthesis, Wiley, New York, 2003; H. Zhao, S. V. Malhotra, Aldrichimica. Acta 2002, 35, 75.

[8] V. Cesar, S. Bellemin-Laponnaz, L. H. Gade, Chem. Soc. Rev., 2004, 33, 619; V. Nair, S. Bindu, V. Sreekumar, Angew. Chem., Int. Ed. 2004, 43, 5130.

[9] Y. Matsuoka, Y. Ishida, D. Sasaki, K. Saigo, Tetrahedron 2006, 62, 8199.

[10] C. A. Vock, C. Scolaro, A. D. Phillips, R. Scopelliti, G. Sawa, P. J. Dyson, J. Med. Chem. 2006, 49, 5552; K. Deka, M. Laskar, J. B. Barnah, Polyhedron 2006, 25, 2525.

[11] I. J. B. Lin, C. S. Vasam, Coord. Chem. Rev. 2007, 251, 642; J. A. Mata, M. Poyatos, E. Peris, Coord. Chem. Rev. 2007, 251, 841. 
[12] G. Mloston, T. Gendek, H. Heimgartner, Helv. Chim. Acta 1998, 81, 1585.

[13] G. Mloston, T. Gendek, H. Heimgartner, Tetrahedron 2000, 56, 5405.

[14] G. Mloston, M. Celeda, G. K. S. Prakash, G. A. Olah, H. Heimgartner, Helv. Chim. Acta 2000, 83, 728.

[15] G. Mloston, M. Jasinski, A. Linden, H. Heimgartner, Helv. Chim. Acta 2006, 89, 1304.

[16] R. C. F. Jones, J. N. Martin, in 'Synthetic Applications of 1,3-Dipolar Cycloaddition Chemistry Toward Heterocycles and Natural Products', Eds. A. Padwa, W. H. Pearson, J. Wiley \& Sons, New York, 2002, p. 1.

[17] R. Loska, M. Makosza, Mendeelev Commun. 2006, 161.

[18] J. Lin,J. Chem, J. Zhav, Y. Zhao, L. Li, H. Zhang, Synthesis 2003, 2661.

[19] H. Cerecetto, A. Gerpe, M. Gonzales, Y. Fernandez Sainz, O. E. Piro, E. E. Castellano, Synthesis 2004, 2678.

[20] H. Lettau, Z. Chem. 1970, 10, 462.

[21] H. Lettau, P. Nuhn, R. Schneider, P. Stenger, Pharmazie 1990, 45, 830.

[22] M. V. Baker, D. H. Brown, B. W. Shelton, A. H. White, J. Chem. Soc., Dalton Trans. 1999, 1483.

[23] a) I. J. Ferguson, K. Schofield, J. Chem. Soc., Perkin Trans. 1 1975, 275; b) R. Bartnik, W. E. Haku, G. Mlostoń, Roczniki Chemii 1977, 51, 49.

[24] C. K. Johnson, 'ORTEP II', Report ORNL-5238, Oak Ridge National Laboratory, Oak Ridge, Tennessee, 1976.

[25] J. Bernstein, R. E. Davis, L. Shimoni, N.-L. Chang, Angew. Chem., Int. Ed. 1995, 34, 1555.

[26] a) W. L. Semon, V. R. Damerell, Org. Synth. 1943, 2, 205; b) W. F. Beech, J. Chem. Soc. 1955, 3095; c) G. B. Bennett, R. B. Mason, Lee J. Alden, J. B. 
Roach Jr., J. Med. Chem. 1978, 21, 627; d) T. Watson, J. Taylor, M. S. Marks, J. Chem. Soc. 1930, 2302.

[27] R. Hooft, KappaCCD Collect Software, Nonius BV, Delft, The Netherlands, 1999.

[28] Z. Otwinowski, W. Minor, in 'Methods in Enzymology', Vol. 276, 'Macromolecular Crystallography', Part A, Eds. C. W. Carter Jr., R. M. Sweet, Academic Press, New York, 1997, p. 307.

[29] A. Altomare, G. Cascarano, C. Giacovazzo, A. Guagliardi, M. C. Burla, G. Polidori, M. Camalli, SIR92, J. Appl. Crystallogr. 1994, 27, 435.

[30] a) E. N. Maslen, A. G. Fox, M. A. O'Keefe, in 'International Tables for Crystallography', Ed. A. J. C. Wilson, Kluwer Academic Publishers, Dordrecht, 1992 Vol. C, Table 6.1.1.1, p. 477; b) D. C. Creagh, W. J. McAuley, in 'International Tables for Crystallography', Ed. A. J. C. Wilson, Kluwer Academic Publishers, Dordrecht, 1992 Vol. C, Table 4.2.6.8, p. 219; c) D. C. Creagh, J. H. Hubbell, in 'International Tables for Crystallography', Ed. A. J. C. Wilson, Kluwer Academic Publishers, Dordrecht, 1992 Vol. C, Table 4.2.4.3, p. 200.

[31] R. F. Stewart, E. R. Davidson, W. T. Simpson, J. Chem. Phys. 1965, 42, 3175.

[32] J. A. Ibers, W. C. Hamilton, Acta Crystallogr. 1964, 17, 781.

[43] G. M. Sheldrick, SHELXL97, Program for the Refinement of Crystal Structures, University of Göttingen, Germany, 1997. 
Legends

Table 1. Imidazole N-Oxides 11 Prepared from $\mathbf{1 0}$ and $\mathbf{1 2}$

Table 2. Prepared Bis-imidazole N-Oxides $\mathbf{1 6}$

Table 3. Crystallographic Data for Compounds $\mathbf{1 1 a}$ and $\mathbf{1 6 b}$

Fig. 1. ORTEP-Plot [24] of the molecular structure of 11a (arbitrary numbering of the atoms, $50 \%$ probability ellipsoids, the $\mathrm{H}_{2} \mathrm{O}$ molecule is not shown)

Fig. 2. ORTEP-Plot [24] of the molecular structure of $\mathbf{1 6 b}$ (arbitrary numbering of the atoms, $50 \%$ probability ellipsoids, the $\mathrm{H}_{2} \mathrm{O}$ molecule is not shown) 
Table 1. Imidazole 3-Oxides 11 Prepared from 10 and 12

\begin{tabular}{|c|c|c|c|c|c|}
\hline 11 & $\mathrm{R}^{1}$ & $\mathrm{R}^{2}$ & $\mathrm{R}^{3}$ & Yield [\%] & M.p. $\left[{ }^{\circ}\right]$ \\
\hline $\mathbf{a}$ & $\mathrm{HO}-\mathrm{CH}_{2}-\mathrm{CH}_{2}-$ & $\mathrm{Me}$ & $\mathrm{Me}$ & 88 & $106-107$ \\
\hline $\mathbf{b}$ & $\mathrm{HO}-\mathrm{CH}_{2}-\mathrm{CH}_{2}-$ & $\mathrm{Ph}$ & $\mathrm{Me}$ & 81 & 186-188 \\
\hline c & $\mathrm{HO}-\mathrm{CH}_{2}-\mathrm{CH}_{2}-$ & $\mathrm{Ph}$ & $\mathrm{Ph}$ & 89 & 197-198 \\
\hline d & $\mathrm{HO}-\mathrm{CH}_{2}-\mathrm{CH}_{2}-$ & $\mathrm{Me}$ & $\mathrm{Ph}$ & 55 & $165-167$ \\
\hline $\mathbf{e}$ & $\mathrm{HO}-\mathrm{CH}_{2}-\mathrm{CH}_{2}-\mathrm{CH}_{2}-$ & $\mathrm{Ph}$ & $\mathrm{Me}$ & 94 & $140-142$ \\
\hline $\mathbf{f}$ & $\mathrm{HO}-\mathrm{CH}_{2}-\mathrm{CH}_{2}-\mathrm{CH}_{2}-$ & $\mathrm{Ph}$ & $\mathrm{Ph}$ & 47 & 191-193 \\
\hline g & $\mathrm{HO}-\mathrm{CH}_{2}-\mathrm{CH}_{2}-\mathrm{CH}_{2}-$ & $\mathrm{Me}$ & $\mathrm{Ph}$ & 66 & $139-140$ \\
\hline h & $\mathrm{Me}-\mathrm{CH}(\mathrm{OH})-\mathrm{CH}_{2}-$ & $\mathrm{Ph}$ & $\mathrm{Me}$ & 90 & $143-145$ \\
\hline i & $\mathrm{Me}-\mathrm{CH}(\mathrm{OH})-\mathrm{CH}_{2}-$ & $\mathrm{Ph}$ & $\mathrm{Ph}$ & 92 & $182-184$ \\
\hline $\mathbf{j}$ & $(S)-\mathrm{Me}-\mathrm{CH}(\mathrm{OH})-\mathrm{CH}_{2}-$ & $\mathrm{Me}$ & $\mathrm{Me}$ & 82 & $119-120$ \\
\hline $\mathbf{k}$ & $\mathrm{HO}-\mathrm{CH}_{2}-\mathrm{CH}(\mathrm{Et})-$ & $\mathrm{Ph}$ & $\mathrm{Me}$ & 86 & $171-174$ \\
\hline 1 & $\mathrm{HO}-\mathrm{CH}_{2}-\mathrm{CH}(\mathrm{Et})-$ & $\mathrm{Ph}$ & $\mathrm{Ph}$ & 71 & 199-200 \\
\hline m & $\begin{array}{l}(S, S)-\mathrm{PhCH}(\mathrm{OH})- \\
\mathrm{CH}\left(\mathrm{CH}_{2} \mathrm{OH}\right)-\end{array}$ & $\mathrm{Ph}$ & $\mathrm{Me}$ & 52 & $177-178$ \\
\hline
\end{tabular}

Table 2. Prepared Bis(imidazole 3-oxides) 16

\begin{tabular}{llllcc}
\hline $\mathbf{1 6}$ & $n$ & $\mathrm{R}^{1}$ & $\mathrm{R}^{2}$ & Yield [\%] & M.p. $\left[^{\circ}\right]$ \\
\hline a & 1 & $\mathrm{H}$ & $\mathrm{Me}$ & 48 & $231-236$ \\
$\mathbf{b}$ & 1 & $\mathrm{H}$ & $\mathrm{Ph}$ & 42 & $236-240$ \\
c & 1 & $\mathrm{Me}$ & $\mathrm{Ph}$ & 35 & $234-237$ \\
d & 2 & $\mathrm{H}$ & $\mathrm{Me}$ & 11 & $191-196$ \\
e & 3 & $\mathrm{H}$ & $\mathrm{Me}$ & 47 & $151-155$ \\
$\mathbf{f}$ & 5 & $\mathrm{H}$ & $\mathrm{Me}$ & 43 & $101-104$ \\
g & 5 & $\mathrm{H}$ & $\mathrm{Ph}$ & 53 & $188-192$ \\
\hline
\end{tabular}


Table 3. Crystallographic Data for Compounds $\mathbf{1 1 a}$ and $\mathbf{1 6 b}$

11a

Crystallized from

Empirical formula

Formula weight

Crystal color, habit

Crystal dimensions [mm]

Temperature [K]

Crystal system

Space group

Z

Reflections for cell determination

$2 \theta$ range for cell determination $\left[{ }^{\circ}\right]$

Unit cell parameters $a[\AA]$

$$
b[\AA]
$$$$
c[\AA]
$$$$
\beta\left[^{\circ}\right]
$$

$D_{x}\left[\mathrm{~g} \mathrm{~cm}^{-3}\right]$

$V\left[\AA^{3}\right]$
$\mathrm{CHCl}_{3} /$ hexane

$\mathrm{C}_{7} \mathrm{H}_{12} \mathrm{~N}_{2} \cdot 0.5 \mathrm{H}_{2} \mathrm{O}$

165.19

colorless, prism

$0.25 \times 0.25 \times 0.25$

$160(1)$

monoclinic

$C 2 / c$

8

2566

4-60

$11.1258(2)$

$11.8321(3)$

12.9472(3)

99.040(2)

1683.22(7)

1.304

0.0993

$\phi$ and $\omega$

60

21719

2460

2006

2459

116; 0

0.0423

0.1154

$0.0542 ; 0.7192$

1.057

$0.009(2)$

0.001

$0.21 ;-0.25$ 16b

$\mathrm{EtOH}$

$\mathrm{C}_{22} \mathrm{H}_{22} \mathrm{~N}_{4} \mathrm{O}_{2} \cdot 2 \mathrm{H}_{2} \mathrm{O}$

410.47

colorless, plate

$0.05 \times 0.17 \times 0.22$

160(1)

orthorhombic

Aba 2

4

1341

4-55

24.0599(8)

10.8504(3)

7.7711(3)

90

2028.7(1)

1.344

0.0941

$\phi$ and $\omega$

55

13330

1248

1127

1248

$145 ; 1$

0.0374

0.0945

$0.0445 ; 1.0001$

1.122

0.001

$0.16 ;-0.20$

a) $w^{-1}=\sigma^{2}\left(F_{\mathrm{o}}^{2}\right)+(a P)^{2}+b P$ where $P=\left(F_{\mathrm{o}}^{2}+2 F_{\mathrm{c}}^{2}\right) / 3$ 
Scheme 1

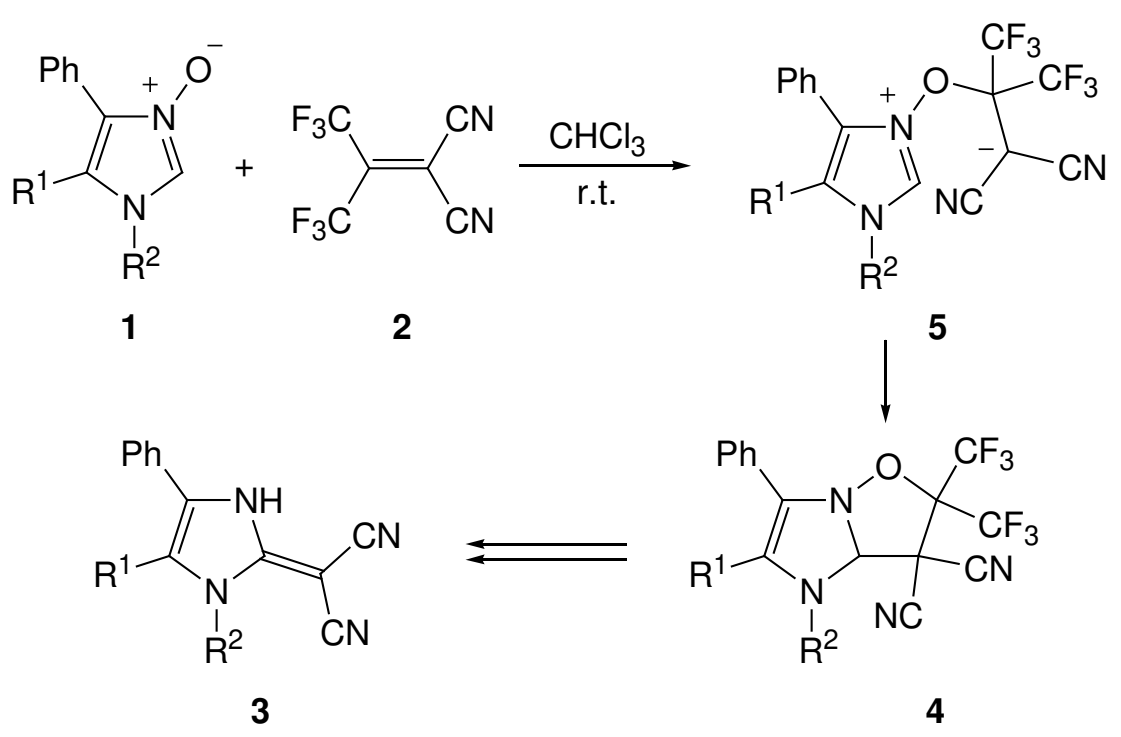

Formulae 6, 7, and 8<smiles>[R]C(CO)n1ccnc1</smiles><smiles>[R]C(O)Cn1ccnc1</smiles><smiles>c1ccc(C(=C(n2ccnc2)n2ccnc2)c2ccccc2)cc1</smiles> 
Scheme 2

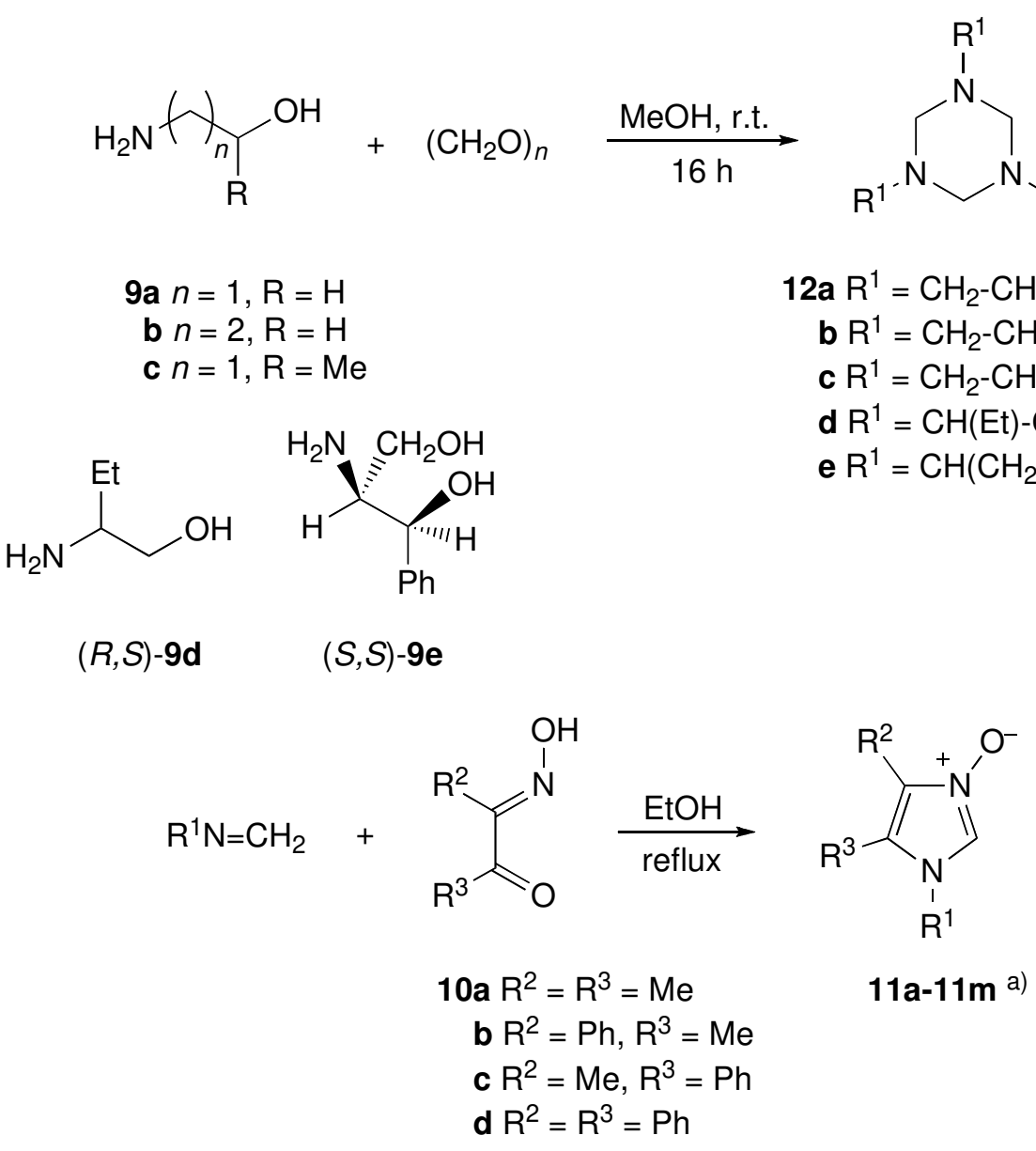

a) See Table 1

Scheme 3<smiles>[R]c1c(-c2ccccc2)[nH]c(=O)n1CCOC(C)=O</smiles>

13a $n=1, \mathrm{R}^{3}=\mathrm{Me}$

b $n=1, \mathrm{R}^{3}=\mathrm{Ph}$

c $n=2, \mathrm{R}^{3}=\mathrm{Me}$<smiles>[R]c1c([R])[n+]([O-])cn1CC(O)CO</smiles>

11

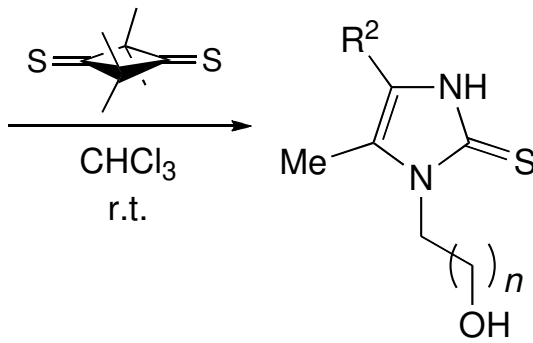

14a $n=1, \mathrm{R}^{2}=\mathrm{Me}$ 
Scheme 4
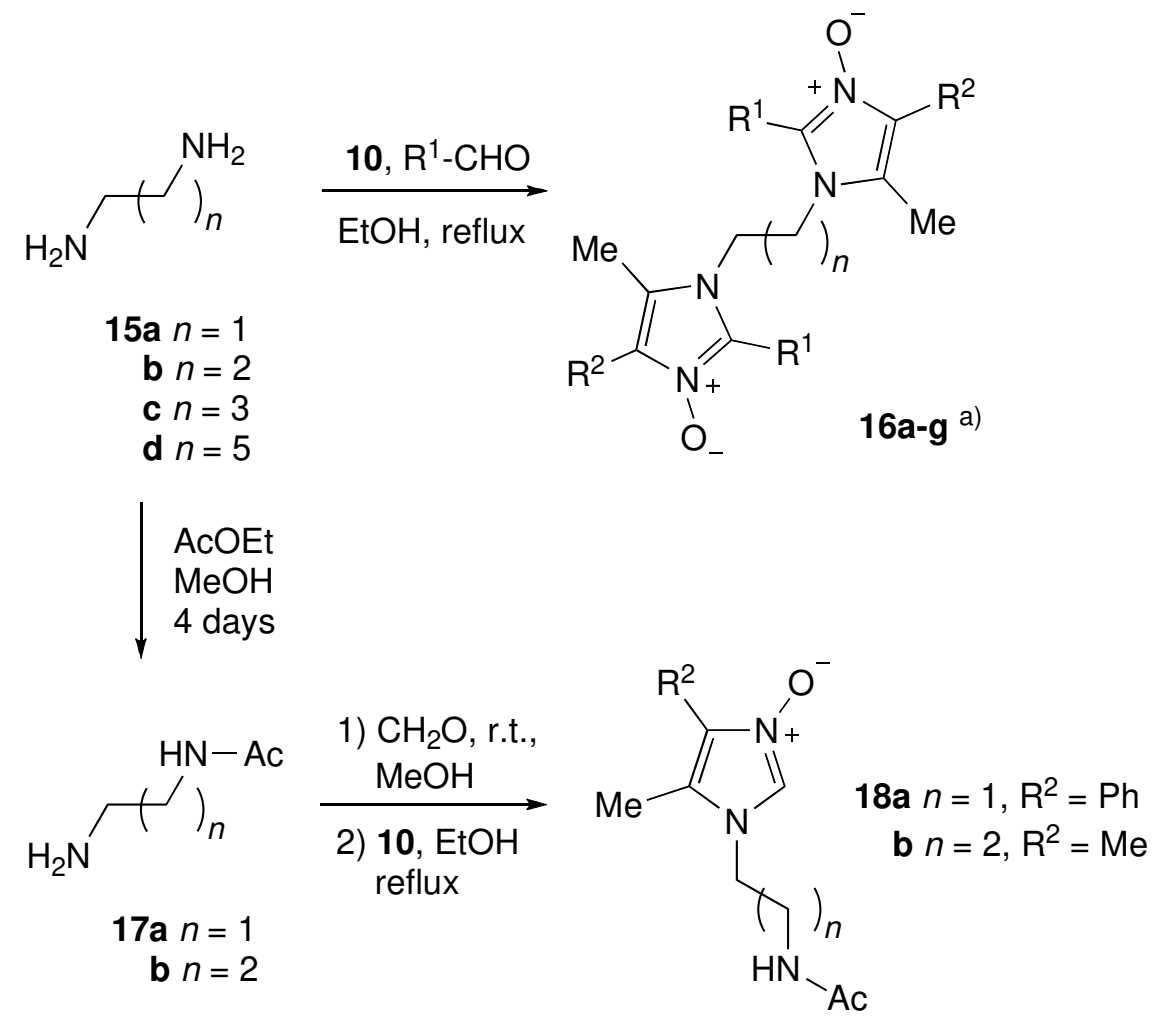

a) See Table 2

Scheme 5

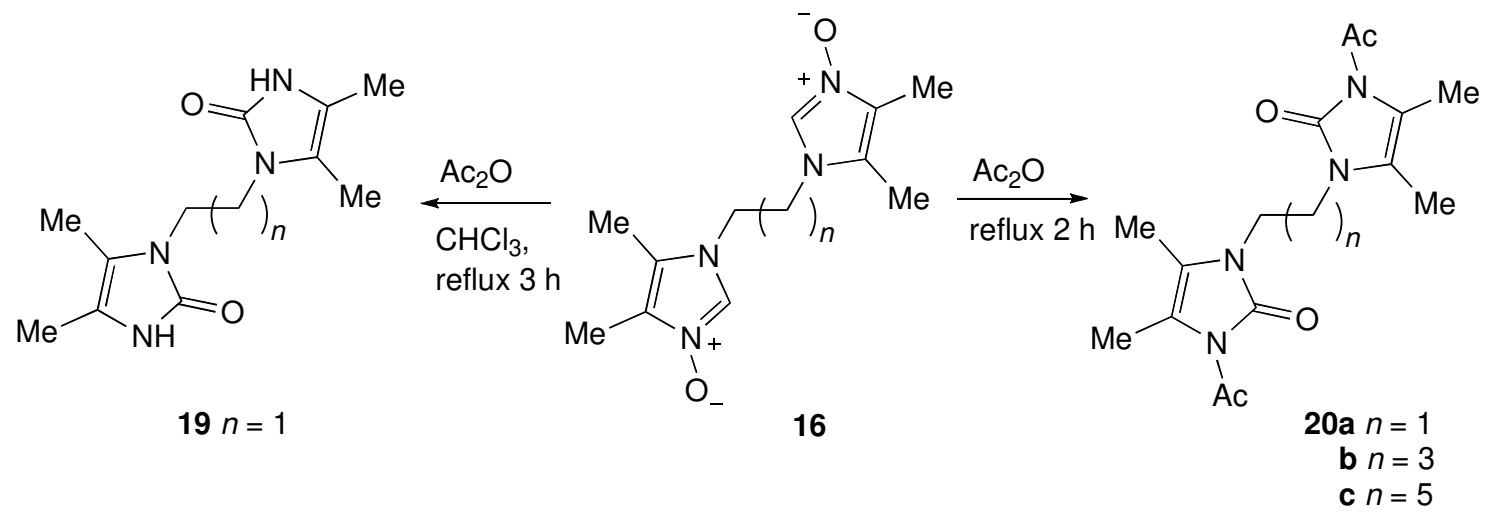


Scheme 6

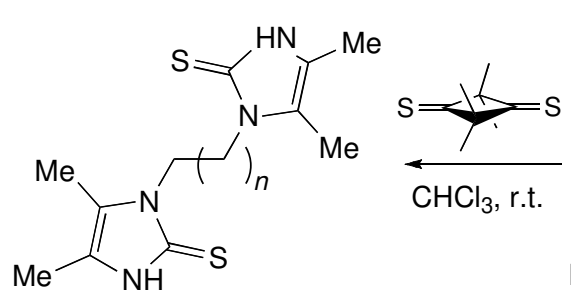

$21 n=3$

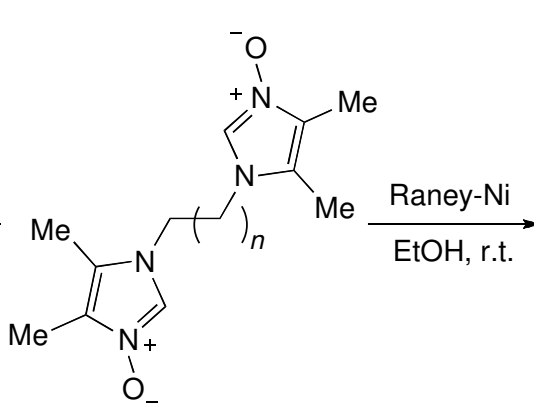

16e $n=3$

f $n=5$

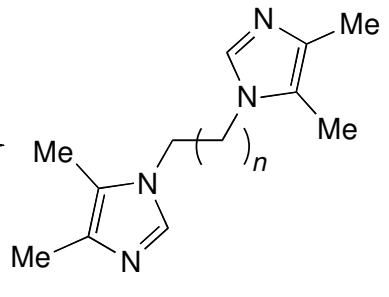

$22 n=5$

Figure 1

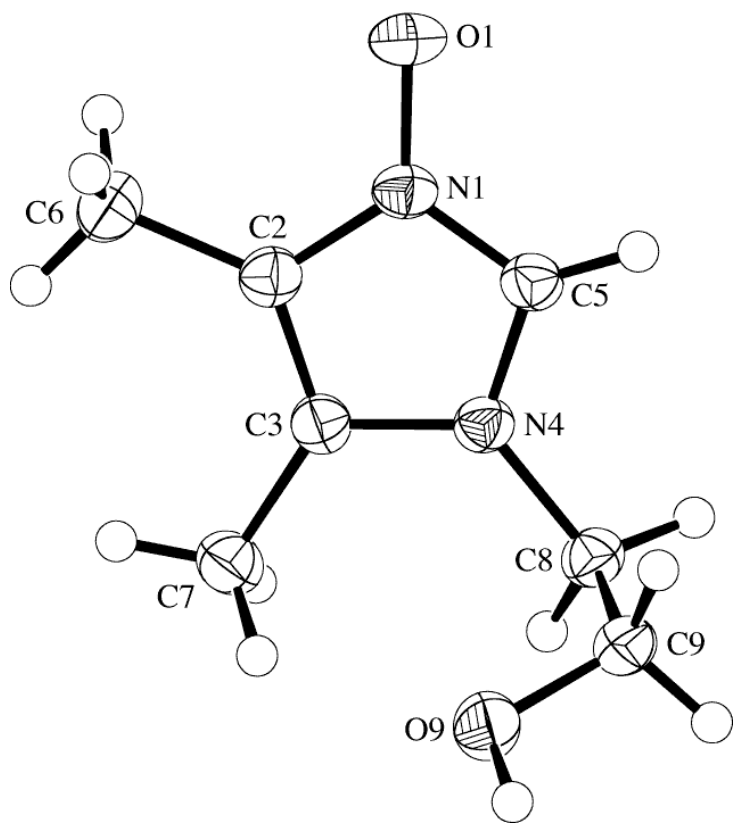


Figure 2

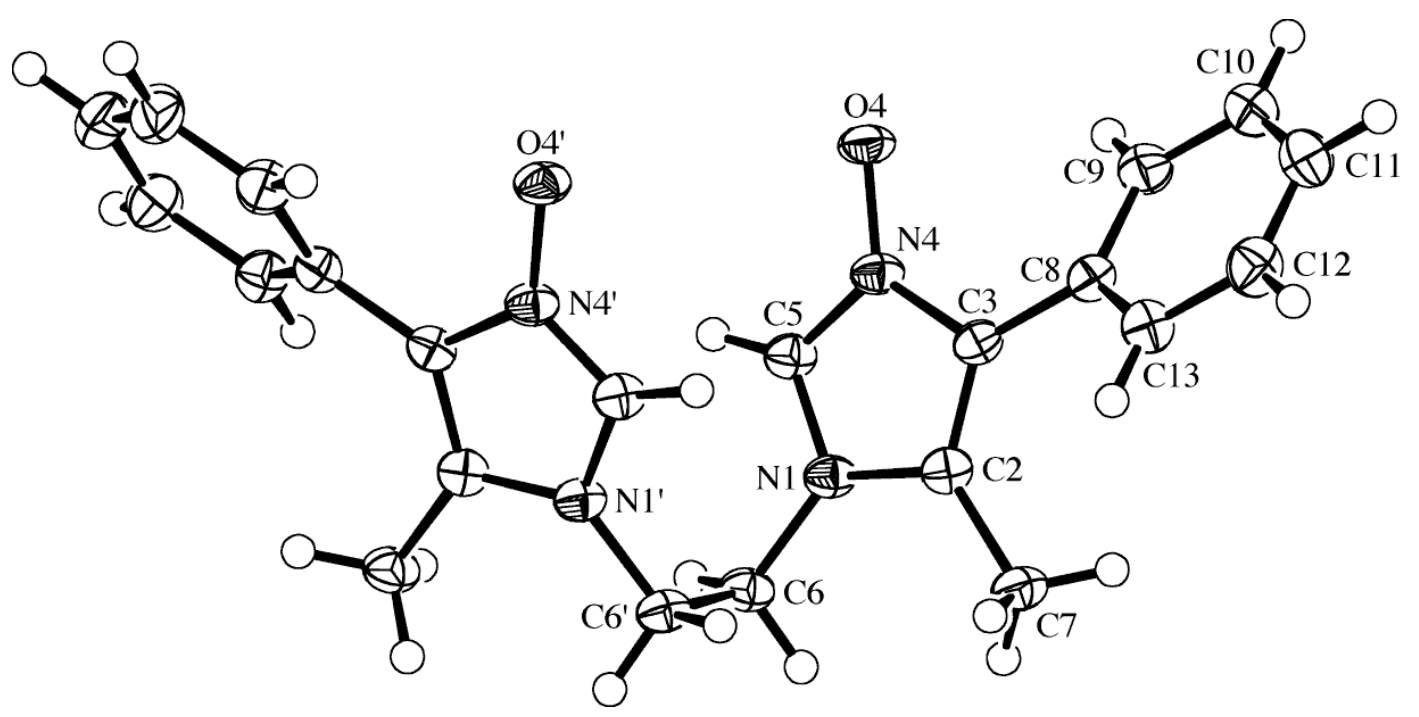




\section{Graphical Abstract}

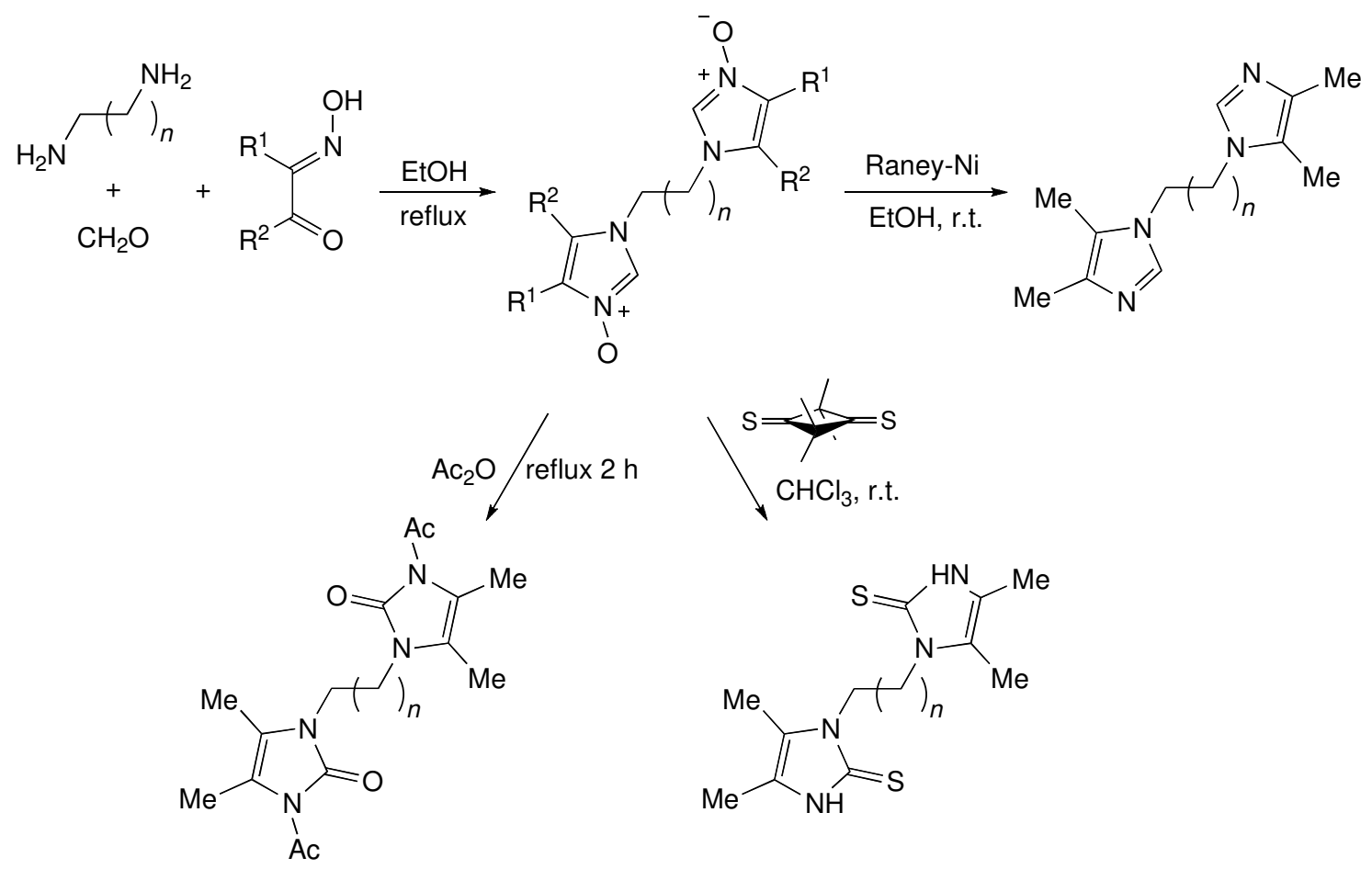

